\title{
Quantization of Poisson-Lie Groups and Applications
}

\author{
Frederic Bidegain, Georges Pinczon \\ Laboratoire d'Algèbre et d'Analyse: Théorie des Représentations, Département de Mathématiques, \\ Université de Bourgogne, B P 138, F-21 004 Dijon Cedex, France \\ e-mail: physmath@satie u-bourgogne.fr
}

Received: 20 July 1995/Accepted: 6 November 1995

\begin{abstract}
Let $G$ be a connected Poisson-Lie group. We discuss aspects of the question of Drinfel'd: can $G$ be quantized? and give some answers. When $G$ is semisimple (a case where the answer is yes), we introduce quantizable Poisson subalgebras of $C^{\infty}(G)$, related to harmonic analysis on $G$; they are a generalization of F.R.T. models of quantum groups, and provide new examples of quantized Poisson algebras.
\end{abstract}

\section{Introduction*}

Quantization in the framework of deformation theory (deformation quantization [26]) was initiated in [2]. The deformation-quantization program of symplectic structures ([2]) leads to various and deep applications in physics and mathematics (e.g. index theory [ 8 and 26]). The existence of quantizations was first proved using some technical assumptions (essentially vanishing of the cohomology group where obstruction sits), and then in full generality: actually, any symplectic Poisson bracket can be quantized ([9 and 26]).

In his Berkeley report [10b], Drinfel'd proposed a similar program of quantization in the case of Poisson-Lie group structures. Here, deformation of algebras will not be enough: one has to deform Hopf algebra structures. In order to quantify the standard Poisson-Lie bracket on simple groups, Drinfel'd introduced deformations of enveloping algebras, which became very popular under the name of quantum groups. These structures contain a lot of combinatorial information, and happened to have fundamental applications (e.g. knot theory [19a and 23]), via their universal $R$-matrix. In [10d], a very natural question was asked: can any Poisson-Lie structure be quantized? This question is, in fact, a multivalued one, since it can be given at least three interpretations, each of which leads to a particular problem, that we shall now state.

\footnotetext{
* Unexplained notations used in this introduction can be found in Sect. 1, Sect 4 (for Poisson algebras, Poisson-Lie groups, etc ) and Appendix A (for representations of semisimple Lie groups)
} 
$\left(\mathbf{P}_{1}\right)$ Problem 1. Given a Poisson-Lie group $G$, does there exists a deformation of the Hopf algebra $C^{\infty}(G)$, with a new product $\star$, unchanged coproduct, and such that:

$$
\text { if } \begin{aligned}
f \star g & =f g+C_{1}(f, g) t+O\left(t^{2}\right), \text { then } \\
\{f, g\} & =C_{1}(f, g)-C_{1}(g, f), \quad \forall f, g \in C^{\infty}(G) ?
\end{aligned}
$$

In this definition, $C^{\infty}(G)$ is considered to be a topological Hopf algebra, and deformation means topological deformation (see [6]). Any solution of $\left(\mathrm{P}_{1}\right)$ will be called a quantization of the Poisson algebra $C^{\infty}(G)$, or simply a quantization of the Poisson-Lie group G.

We recall that deformations with unchanged coproduct are called preferred deformations ([13]). This condition is imposed to quantization because of the invariance property of the Poisson-Lie bracket:

$$
\delta(\{f, g\})=\{\delta(f), \delta(g)\}, \quad \forall f, g \in C^{\infty}(G) .
$$

It is therefore natural to impose the same compatibility of the $\star$-product with respect to the coproduct of $C^{\infty}(G)$, i.e.:

$$
\delta(f \star g)=\delta(f) \star \delta(g), \quad \forall f, g \in C^{\infty}(G) .
$$

$\left(\mathbf{P}_{2}\right)$ Problem 2. Given a finite dimensional Lie bialgebra $(\mathfrak{g}, \rho)$, does there exist a deformation of the Hopf algebra $\mathscr{U}(\mathfrak{g})$, with new coproduct $\widetilde{\Delta}$, such that:

$$
\left.\left(\widetilde{\Delta}-\widetilde{\Delta}^{\mathrm{op}}\right)\right|_{\mathrm{g}}=\rho t+O\left(t^{2}\right) ?
$$

Once more, deformation has to be taken in the topological sense, $\mathscr{U}(\mathfrak{g})$ being endowed with its natural topology ([6]). Any solution of $\left(\mathrm{P}_{2}\right)$ will be called $a$ quantization of the Lie bialgebra $(\mathfrak{g}, \rho)$, or a quantized enveloping algebra.

Relations between $\left(\mathrm{P}_{1}\right)$ and $\left(\mathrm{P}_{2}\right)$ are not obvious, though it is clear that a natural choice for $(\mathfrak{g}, \rho)$ is the Lie bialgebra structure defined from the Poisson-Lie structure of $G$. Very heuristically (but it can be made rigorous in the present context as we shall show later on), considering that $\mathscr{U}(\mathfrak{g})$ and $C^{\infty}(G)$ are related by some duality, and given a solution of $\left(\mathrm{P}_{2}\right)$, transposition of the new Hopf structure of $\mathscr{U}(\mathfrak{g})$ should lead to a new Hopf structure on $C^{\infty}(G)$. But, if the product of $\mathscr{U}(\mathfrak{g})$ is changed, we shall not obtain a preferred deformation, so invariance with respect to $\delta$ will be lost. Therefore, we have to ask more:

$\left(\mathbf{P}_{3}\right)$ Problem 3. Given a finite dimensional Lie bialgebra $(\mathfrak{g}, \rho)$, does there exist a solution of $\left(\mathrm{P}_{2}\right)$ with unchanged product?

Any solution of $\left(\mathrm{P}_{3}\right)$ will be called a preferred quantization of the Lie bialgebra $(\mathfrak{g}, \rho)$.

Let us briefly recall what was known up to now. If $G=\mathbb{R}^{n},\left(\mathrm{P}_{1}\right)$ is solved by the Poincaré-Birkhoff-Witt Theorem ([15]). For triangular bialgebras (i.e. such that $\rho(X)=[\Delta(X), r], \forall X \in \mathfrak{g}$, with $r \in \mathfrak{g} \wedge \mathfrak{g}$, a solution of the classical YangBaxter equation), it was shown that $\left(\mathrm{P}_{1}\right)$ and $\left(\mathrm{P}_{3}\right)$ can be solved in the simply connected case ([10a]). Introducing quantum groups, Drinfel'd was able to solve $\left(\mathrm{P}_{3}\right)$ for the standard bialgebra structure on semisimple $\mathfrak{g}$; topological deformation theory was then used in [5 and 6] to solve $\left(\mathrm{P}_{1}\right)$ in that case. After several attempts (e.g. [19b]), it was shown that $\left(\mathrm{P}_{2}\right)$ can be solved for any finite dimensional 
Lie bialgebra [11]. The main problems, i.e. $\left(\mathrm{P}_{3}\right)$ and especially $\left(\mathrm{P}_{1}\right)$, are still unsolved, up to now, for general $\mathfrak{g}$, or $G$.

Furthermore, let us mention another kind of quantization, the so-called FaddeevReshetikhin-Takhtajan models (or F.R.T. models) [12]. It works for simple classical matrix groups. Actually, it was proved in [5 and 6], that F.R.T. quantization is a quantization, in the sense of $\left(\mathrm{P}_{1}\right)$, of the Hopf algebra of coefficients of finitedimensional $(f . d$.) representations of $G$ (this was expected by several authors, who gave various heuristic proofs). Even more, the above-mentioned solution of $\left(\mathrm{P}_{1}\right)$, in that case, can be restricted to f.d. coefficients, and one recovers F.R.T. models ([5 and 6]). For a semisimple $G$, f.d. coefficients are dense in $C^{\infty}(G)$ if and only if $G$ is linear ([5]); so, for a linear $G$, the $\star$-quantization is specified by its restriction to f.d. coefficients. This is what we shall call a F.R.T. core: a $\star$-stable dense subalgebra of $C^{\infty}(G)$, the first example being exactly F.R.T. models. It is a very interesting (and completely unexplored up to now) problem to find F.R.T. cores, for instance for nonlinear $G$; $\star$-stable subalgebras are also of interest, because they provide ipso facto new examples of Poisson-Lie algebras, and of their quantization.

So, we have two objectives in the present paper:

- first, to go further in the resolution of $\left(\mathrm{P}_{1}\right)$,

- second, to construct new F.R.T. cores, and examples of $\star$-stable subalgebras, in relation to representation theory and harmonic analysis.

Now, we describe our main results.

We prove a general statement concerning the resolution of $\left(\mathrm{P}_{1}\right)$, when $\left(\mathrm{P}_{3}\right)$ can be solved:

Theorem 1 (6.1.1). Let $G$ be a simply connected Poisson-Lie group. If $\left(\mathrm{P}_{3}\right)$ can be solved, then $\left(\mathrm{P}_{1}\right)$ can be solved.

Our proof gives an algorithm for the computation of the solution. We also show that the cochains of the obtained $\star$-product on $C^{\infty}(G)$ are bidifferential operators; they are of finite order.

In the case of semisimple groups, a well-known argument of Drinfel'd shows that $\left(\mathrm{P}_{2}\right)$ and $\left(\mathrm{P}_{3}\right)$ are equivalent problems. Using [11], $\left(\mathrm{P}_{2}\right)$ can be solved, then an easy application of arguments developed in [5 and 6] gives:

Theorem 2 (4.3.2). Let $G$ be a connected semisimple Poisson-Lie group. Then $\left(\mathrm{P}_{1}\right)$ can be solved.

Note that simple connectedness is not needed in Theorem 2. We show that, in the semisimple case, the cochains are bidifferential operators of finite order.

Using [11], where $\left(\mathrm{P}_{3}\right)$ is proved in that case, the case of "double groups" is very similar:

Theorem 3 (4.3.3.3). Let $G$ be a connected Poisson-Lie group. We assume that the associated Lie bialgebra is the double of some Lie bialgebra. Then $\left(\mathrm{P}_{1}\right)$ can be solved.

In that case also, the cochains are bidifferential operators of finite order.

Let us restrict our attention, in the sequel, to connected semisimple Poisson-Lie groups, with finite center. For such groups, it was shown by Drinfel'd [10c] that solutions of $\left(\mathrm{P}_{3}\right)$ are always obtained from the initial coproduct by a twist. As a consequence, we are able to construct $\star$-stable subalgebras and F.R.T. cores using 
representation theory, and coefficients, not only of finite dimensional representations, but also of infinite-dimensional ones:

Theorem 4 (5.3.4) \& (3.3.1).

1. Let $\Pi$ be a family of $C^{\infty}$ representations of $G$, and $\mathbb{C}[\Pi]$ the subalgebra of $C^{\infty}(G)$ generated by their coefficients. Then $\mathbb{C}[\Pi]$ is $\star$-stable.

2. Let $\Pi$ be a family of admissible Banach representations of $G, K$ be a maximal compact subgroup of $G$ and $\mathbb{C}_{K}[\Pi]$ the subalgebra of $C^{\omega}(G)$ generated by their admissible coefficients. Then $\mathbb{C}_{K}[\Pi]$ is $\star$-stable.

3. In case 1 (resp. 2), if $\Pi$ is conjugation-stable and contains sufficiently many representations, then $\mathbb{C}[\Pi]$ (resp. $\mathbb{C}_{K}[\Pi]$ ) is a F.R.T. core.

(5.3.1) shows that $C^{\omega}(G)$ is $\star$-stable. Note that $\mathbb{C}_{K}[\Pi]$ is a domain since it is contained in $C^{\omega}(G)$. In Theorem 4 , one can take, for instance, the family $\Pi_{i f}$ of the irreducible f.d. representations of $G$; then $\mathbb{C}\left[\Pi_{i f}\right]=\mathbb{C}_{K}\left[\Pi_{i f}\right]=\operatorname{Span}\left[\operatorname{Coeff}\left[\Pi_{i f}\right)\right]$, and $\mathbb{C}\left[\Pi_{i f}\right]$ is a F.R.T. core if and only if $G$ is linear ([5]). For general $G$, F.R.T. cores always exist, but infinite-dimensional representations are needed:

Theorem 5 (5.3.6). There exist unitary irreducible representations $\pi_{1}, \ldots, \pi_{n}$ of $G$ such that $\mathbb{C}_{K}\left[\pi_{1}, \ldots, \pi_{n}\right]$ is a F.R.T. core. If $G$ is not compact, $\pi_{1}, \ldots, \pi_{n}$ can be constructed using the unitary principal series induction techniques.

The first claim of Theorem 5 is the generalisation of a similar result valid for compact groups ([6]). There is some freedom in the choice of $\pi_{1}, \ldots, \pi_{n}$.

Let us now restrict to the case when $\operatorname{rank} G=\operatorname{rank} K$. This ensures existence of discrete series, but we want more, since we want to have holomorphic discrete series. So we assume that, once a compact Cartan subalgebra $\mathfrak{h}$ is fixed, there exists a Borel subalgebra $\mathfrak{b} \supset \mathfrak{h}$, and nontrivial $\mathfrak{b}$-extreme unitary representations of $G$ (these representations were introduced by Harish-Chandra in his early work on discrete series [16]). We denote by $\Pi_{\mathfrak{b}}$ the set of $\mathfrak{b}$-extreme unitary representations, and obtain:

Theorem 6 (5.4.1). $\operatorname{Coeff}_{K}\left(\Pi_{\mathfrak{b}}\right)$ is a $a$-stable subalgebra of $C^{\omega}(G)$. One has:

$$
\operatorname{Coeff}_{K}\left(\Pi_{\mathfrak{b}}\right)=\bigoplus_{\pi \in \Pi_{\mathfrak{b}}} \operatorname{Coeff}_{K}(\pi)
$$

If $\Pi_{\mathfrak{b}}^{1}$ is the subset of integrable elements in $\Pi_{\mathfrak{b}}$, and $\Pi_{\mathfrak{b}}^{2}$ the subset of squareintegrable elements, then:

$$
\operatorname{Coeff}_{K}\left(\Pi_{\mathfrak{b}}^{1}\right) \subset \operatorname{Coeff}_{K}\left(\Pi_{\mathfrak{b}}^{2}\right), \quad \text { and } \quad \operatorname{Coeff}_{K}\left(\Pi_{\mathfrak{b}}^{2}\right) \star \operatorname{Coeff}_{K}\left(\Pi_{\mathfrak{b}}^{2}\right) \subset \operatorname{Coeff}_{K}\left(\Pi_{\mathfrak{b}}^{1}\right)[[t]] .
$$

So, for any choice of an "admissible" Borel subalgebra, there is a corresponding Poisson algebra of coefficients of unitary $\mathfrak{b}$-extreme representations, and $\star$ is $a$ quantization of this Poisson algebra.

Theorem 6 is a consequence of the reduction of any tensor product of elements of $\Pi_{\mathfrak{b}}$ into direct sum of elements of $\Pi_{\mathfrak{b}} \quad((3.5 .4))$. It is a generalisation of the results of [4], who treated the case of $G=\mathbf{S U}(1,1)$.

Theorems 1 to 6 are, in our opinion, the main results of the paper; nevertheless, there are several other results or remarks which can be of interest by themselves: for instance, we show in Sect. 7 that the existence of a solution for $\left(\mathrm{P}_{2}\right)$ can be interpreted as a "free quantization up to flat functions." These results are not listed in this introduction, and have to be found in the sections of the paper. 
We tried to write a self-contained exposition; so we often recall known results, when needed. Nevertheless, it would be too long to recall the complete theory of topological Hopf algebras and their deformations, including well-behaved Hopf algebras, which is developed in [6]. This machinery being an essential tool in the present paper, we recommend that the reader consult [6].

Let us describe the sections of the paper:

Section 1 contains all general material and notations about the well-behaved Hopf algebra $C^{\infty}(G)$ and its strong dual $A(G)$. This presentation is not so usual, but very convenient, so we give some details.

Section 2 deals with subalgebras of $C^{\infty}$ and $C^{\omega}$ coefficients, Sect. 3 with subalgebras of admissible coefficients (for semisimple $G$ ), in the classical case, i.e. before quantization. Subalgebras defined from $\mathfrak{b}$-extreme unitary representations are studied at the end of the section, the example of $G=\mathbf{S U}(1,1)$ is presented in detail.

In Sect. 4, we recall, for convenience, the basic notations concerning quantization of Poisson algebras. We then discuss the semisimple case, and prove Theorem 2. We obtain Theorem 3 by similar arguments.

In Sect. 5, we specialize to connected semisimple Poisson-Lie groups, with finite center. Drinfel'd's twist formula provides explicit formulæ for the computation of product of coefficients (5.2.1). Using the results of Sects. 2,3 and 4, and Appendix B, we prove Theorems 4,5 and 6 .

In Sect. 6, we prove Theorem 1. The proof uses integrability results on deformations of representations obtained in [18], that we recall at the beginning of the section.

In Sect. 7 , we interpret the solutions $\left(\mathrm{P}_{2}\right)$ as free quantizations up to flat functions, using the duality between the space of formal Taylor series and the enveloping algebra.

Apart from Theorems 1 and 3 (and Sects. 6 and 7), most of the paper deals with semisimple Lie groups, and their representations. Since the theory of representations of semisimple has developed a jungle-like and often author-dependent terminology, we give in Appendix A, a complete list of the vocabulary and results needed in the paper, for the convenience of the reader.

Finally in the paper, we use a $C^{\infty}$ Stone-Weierstrass Theorem, adapted to Lie groups, which is useful:

$C^{\infty}$ Stone-Weierstrass Theorem. Let $G$ be a connected Lie group, $\mathscr{S}$ a closed subalgebra of $C^{\infty}(G)$ stable under conjugation, containing sufficiently many functions to separate between points of $G$, and stable under the left or right regular representation of $G$. Then $\mathscr{S}=C^{\infty}(G)$.

Since we have not been able to find a reference for this theorem, we give a proof in Appendix B.

\section{Notations}

1.1. All vector spaces used in this paper are complex vector spaces, all Lie groups are real Lie groups, all algebras have a unit element. A t.v.s. is a complete, Hausdorff and locally convex, topological vector space; if $V_{1}$ and $V_{2}$ are t.v.s., $\mathscr{L}\left(V_{1} ; V_{2}\right)$ is the space of continuous linear mappings from $V_{1}$ into $V_{2}, V^{*}$ is the strong dual of $V$. The tensor product of t.v.s. is the topological projective one, denoted by $\widehat{\otimes}$. 
In the paper, we need several results partly from geometry (essentially Lie group theory), partly from t.v.s. theory (distribution theory), partly from Hopf algebra theory. All these results are well-known, but not presented all together in the same textbook, so we shall recall them in detail. For the proofs, we refer to $[7,14,17,22,25]$, etc. The Hopf algebra theory we use is topological Hopf algebra theory, introduced and developed in [6]: the underlying vector spaces are t.v.s., the tensor product is $\widehat{\otimes}$, and all the usual operations of Hopf algebras are supposed to be continuous. Any usual (i.e. in the algebraic sense) Hopf algebra of countable dimension is a topological Hopf algebra, when endowed with its natural topology. A (topological) Hopf algebra is well-behaved ([6]), if the underlying t.v.s. is Fréchet or DF, and nuclear. If $\mathfrak{U}$ is a well-behaved Hopf algebra, then (by transposition) $\mathfrak{U}^{*}$ is also a well-behaved Hopf algebra; due to the reflexivity of such spaces, $\mathfrak{U}^{* *}=\mathfrak{U}$, as Hopf algebras. For more details, see [6].

1.2. Given a connected Lie group $G$, we denote by $e$ the identity element of $G$, by $\mathfrak{g}_{0}$ its (real) Lie algebra, by $\mathfrak{g}$ the complexification of $\mathfrak{g}_{0}$, and by $\mathscr{U}(\mathfrak{g})$, or $\mathscr{U}$, the enveloping algebra of $\mathfrak{g}$. The space $C^{\infty}(G)$ of the $C^{\infty}$ maps from $G$ into $\mathbb{C}$, with its usual Fréchet topology, is a well-behaved Hopf algebra, with the product the Abelian product of functions, the coproduct $\delta: C^{\infty}(G) \rightarrow C^{\infty}(G) \widehat{\otimes} C^{\infty}(G)=$ $C^{\infty}(G \times G)$ defined by $\delta(f)(x, y)=f(x y)$, the antipode $S(f)(x)=f\left(x^{-1}\right)$, and the counit $\varepsilon(f)=f(e)$. Transposition of the Hopf structure of $C^{\infty}(G)$ to $A(G)=$ $C^{\infty}(G)^{*}$ provides a well-behaved Hopf algebra structure on $A(G)$ : the elements of $A(G)$ are the compactly-supported distributions, and the product is the convolution product, that we shall denote by $S \cdot T(S, T \in A(G))$ or simply by $S T$, if there is no ambiguity. The Dirac map $\partial: G \rightarrow A(G)$, mapping $x$ to the Dirac measure on $x\left(\partial_{x}\right)$, is a homeomorphism from $G$ onto the closed subset of group-like elements of $A(G)$. Moreover, one has $\partial_{x} \cdot \partial_{y}=\partial_{x y}$, and $\partial_{e}$ is the unit of $A(G)$, so it is natural to identify $G$ and $\partial_{G}$, which we assume is done in the sequel. One has $\overline{\operatorname{Span}(G)}=A(G)$. Then, the fundamental property of $A(G)$ is that any $C^{\infty}$ map $f$ from $G$ into a t.v.s. $V$ can be extended to a continuous linear $f: A(G) \rightarrow V$. This is a consequence of nuclearity: one has

$$
C^{\infty}(G ; V)=C^{\infty}(G) \widehat{\otimes} V=A(G)^{*} \widehat{\otimes} V=\mathscr{L}(A(G) ; V)
$$

For instance, any $C^{\infty}$ representation $\pi$ of $G$ in a t.v.s. $V$ lifts to a representation of $A(G)$ on $V$ (see Appendix A).

1.3. We can identify $\mathscr{U}(\mathfrak{g})$ and the algebra of the left invariant differential operators on $G$. Then we define a linear one-to-one map $i: \mathscr{U}(\mathfrak{g}) \rightarrow A(G)$ by:

$$
\langle f \mid i(X)\rangle=\left\langle X(f) \mid \partial_{e}\right\rangle, \quad \forall X \in \mathscr{U}(\mathfrak{g}), \forall f \in C^{\infty}(G) .
$$

$i$ is a one-to-one morphism of algebras from $\mathscr{U}(\mathfrak{g})$ onto $i(\mathscr{U}(\mathfrak{g}))$, so we identify both, and consider that $\mathscr{U}(\mathfrak{g}) \subset A(G) . \mathscr{U}(\mathfrak{g})$ is weakly closed, since it is exactly the orthogonal of the space of $C^{\infty}$ functions which are flat at $e$, and $A(G)$ induces on $\mathscr{U}(\mathfrak{g})$ the finest t.v.s. topology, i.e. the natural topology, as defined in [6]. One has:

$$
\langle f \mid X\rangle=\left.\frac{\mathrm{d}}{\mathrm{d} \tau}\langle f \mid \exp \tau X\rangle\right|_{\tau=0}, \quad \forall X \in \mathfrak{g}_{0}, \forall f \in C^{\infty}(G),
$$


so $\left.\frac{\mathrm{d}}{\mathrm{d} \tau} \exp \tau X\right|_{\tau=0}=X$, in $A(G)$, for $X \in \mathfrak{g}_{0}$. As a consequence:

$$
\frac{\mathrm{d}}{\mathrm{d} \tau} \exp \tau X=X \cdot \exp \tau X=\exp \tau X \cdot X
$$

in $A(G)$, for $X \in \mathfrak{g}_{0}$.

The Hopf structure of $A(G)$ is exactly the extension of the usual Hopf structure of $\mathscr{U}$, i.e., if $\Delta$ is the coproduct of $A(G)$, one has, $\forall X \in \mathfrak{g}, \Delta(X)=X \otimes 1+1 \otimes X$, $S(X)=-X$, and the counit $\varepsilon$ is the trivial representation of $\mathfrak{g}$.

1.4. Given a $C^{\infty}$ representation $\pi$ of $G$ in a t.v.s. $V$, as mentioned in Appendix A, it extends to a representation of $A(G)$, and, as a consequence of the formula (1.A), the restriction of $\pi$ to $\mathscr{U}(\mathfrak{g}) \subset A(G)$ is exactly what is usually called the differential of $\pi$.

For instance, the left and right regular representations of $G$ on $C^{\infty}(G)$ are representations of $A(G)$, defined by the formulae:

$$
\begin{aligned}
& \langle\boldsymbol{L}(S) f \mid T\rangle=\langle f \mid S(S) \cdot T\rangle, \quad \forall f \in C^{\infty}(G), \forall S, T \in A(G) . \\
& \langle\boldsymbol{R}(S) f \mid T\rangle=\langle f \mid T \cdot S\rangle,
\end{aligned}
$$

Given $X \in \mathscr{U}(\mathfrak{g}), \boldsymbol{R}(X)$ is exactly the left invariant differential operator corresponding to $X$, and $\boldsymbol{L}(X)$ is exactly the right invariant differential operator corresponding to $X$ :

$$
\begin{aligned}
& {[\boldsymbol{L}(X) f](x)=\langle f \mid S(X) \cdot x\rangle, \quad \forall f \in C^{\infty}(G), \forall x \in G, \forall X \in \mathscr{U}(\mathfrak{g}) .} \\
& {[\boldsymbol{R}(X) f](x)=\langle f \mid x \cdot X\rangle,}
\end{aligned}
$$

Contragredient $\check{\boldsymbol{L}}$ and $\check{\boldsymbol{R}}$ are $C^{\infty}$ representations, when extended to $A(G)$, they are given by:

$$
\begin{aligned}
\check{\boldsymbol{L}}(S) T & =S \cdot T, \\
\check{\boldsymbol{R}}(S) T & =T \cdot S(S), \quad \forall S, T \in A(G) .
\end{aligned}
$$

1.5. The Adjoint representation of $G$ on $A(G)$ is the $C^{\infty}$ representation defined by:

$$
\operatorname{Ad}(x) S=x \cdot S \cdot x^{-1}, \quad \forall x \in G, \forall S \in A(G) .
$$

It extends to a representation, still denoted $\mathrm{Ad}$, of $A(G)$ on itself.

One has $\operatorname{Ad}(X) Y=[X, Y], \forall X, Y \in \mathfrak{g}$, so $\left.\operatorname{Ad}\right|_{\mathfrak{g}}$ acting on $\mathfrak{g}$ is what is usually called the adjoint representation of $\mathfrak{g}$. As for $\left.\mathrm{Ad}\right|_{G}$ acting on $\mathfrak{g}$, it is the usual Adjoint representation of $G$ on $\mathfrak{g}$. Let $\mathscr{U}_{n}, n \geqq 0$, be the canonical filtration of $\mathscr{U}(\mathfrak{g})$, then $\operatorname{Ad}(x) \mathscr{U}_{n} \subset \mathscr{U}_{n}, \forall x \in G$, so each $\mathscr{U}_{n}$ (and consequently $\mathscr{U}(\mathfrak{g})$ ) is a stable subspace for the Adjoint representation of $A(G)$.

1.6. Finally, let us give some precise information about the notion of the differential operators on $C^{\infty}(G)$. They are defined from $\mathscr{U}(\mathfrak{g})$-valued $C^{\infty}$ functions from $G$ to $A(G)$ : Given such a function $d$, the associated differential associated operator is $D: C^{\infty}(G) \rightarrow C^{\infty}(G):$

$$
\langle D f \mid x\rangle=\langle f \mid S(d(x)) \cdot x\rangle=\left\langle f \mid x \cdot \operatorname{Ad}\left(x^{-1}\right)[S(d(x))]\right\rangle, \quad \forall f \in C^{\infty}(G), \quad \forall x \in G .
$$


For instance, for constant functions, one recovers the left regular representation of $\mathscr{U}(\mathfrak{g})$ in $C^{\infty}(G),[\boldsymbol{L}(X) f](x)=\langle f \mid S(X) \cdot x\rangle,\left(\forall f \in C^{\infty}(G), \forall x \in G, \forall X \in \mathscr{U}(\mathfrak{g})\right)$. The right regular representation of $\mathscr{U}(\mathfrak{g})$ occurs for functions of type $x \mapsto \operatorname{Ad}(x) X$ $(X \in \mathscr{U}(\mathfrak{g})):[\boldsymbol{R}(X) f](x)=\langle f \mid x \cdot X\rangle=\langle f \mid[\operatorname{Ad}(x) X] \cdot x\rangle,\left(\forall f \in C^{\infty}(G), \forall x \in G\right)$.

A differential operator is of finite order if it comes from a $C^{\infty} \mathscr{U}_{n}$-valued function, for a fixed $n$, from $G$ into $A(G)$. For instance, the left and right regular representations of $\mathscr{U}(\mathfrak{g})$ in $C^{\infty}(G)$ are representations by differential operators of finite order.

The $\star$-products are generally expected to have bidifferential operators as cochains. The notion of bidifferential operators is a straightforward improvement: one takes $(\mathscr{U} \otimes \mathscr{U})$-valued $C^{\infty}$ functions from $G$ into $A(G) \widehat{\otimes} A(G)=A(G \times G)$, and given such a function $d$, one defines $D$ by, $\forall f, g \in C^{\infty}(G), \forall x \in G$ :

$$
\begin{aligned}
\langle D(f, g) \mid x\rangle & =\left\langle f \otimes g \mid S^{(2)}(d(x)) \cdot(x \otimes x)\right\rangle \\
& =\left\langle f \otimes g \mid(x \otimes x) \cdot \operatorname{Ad}^{(2)}\left(x^{-1} \otimes x^{-1}\right)\left[S^{(2)}(d(x))\right]\right\rangle,
\end{aligned}
$$

where $S^{(2)}$ is the antipode of $A(G \times G), \mathrm{Ad}^{(2)}$ the Adjoint representation of $G \times G$. A finite order bidifferential operator comes from a $(\mathscr{U} \otimes \mathscr{U})_{n}$-valued function, for a fixed $n$, from $G$ into $A(G \times G)$.

This is the end of the list of general notations we need. Specific notations for representations of semisimple Lie groups are given in Appendix A.

\section{2. $C^{\infty}$ and $C^{\omega}$ Coefficients}

2.1. Let $V$ be a t.v.s., $\mathscr{L}(V)$ the algebra of continuous linear maps from $V$ into $V$ and $\mathscr{L}_{0}(V)$ the ideal of finite rank continuous maps, i.e. those $f \in \mathscr{L}(V)$ such that $\operatorname{dim}(\operatorname{Im} f)<\infty$.

\subsubsection{Lemma. Let $V^{*}$ be the topological dual of $V$. Then $\mathscr{L}_{0}(V)=V^{*} \otimes V$.}

Proof. Obviously, $V^{*} \otimes V$ is contained in $\mathscr{L}_{0}(V)$ if one defines, for $\varphi \in V^{*}$, $\mathbf{v} \in V: \mathbf{w} \in V,(\varphi \otimes \mathbf{v})(\mathbf{w})=\varphi(\mathbf{w}) \mathbf{v}$.

Conversely, if $f \in \mathscr{L}_{0}(V)$, then $\operatorname{Ker} f$ is of finite codimension, so it has a topological supplementary $S$. Let us introduce the projection $p$ onto this supplementary with respect to the reduction $V=S \oplus \operatorname{Ker} f$. Then $S^{*} \subset V^{*}$, since any element $\varphi \in S^{*}$ can be extended to $\tilde{\varphi}=\varphi \circ p \in V^{*}$. Now, let $\left\{s_{1}, \ldots, s_{n}\right\}$ be a basis of $S,\left\{\varphi_{1}, \ldots, \varphi_{n}\right\}$ its dual basis and $\left\{\mathbf{v}_{1}, \ldots, \mathbf{v}_{r}\right\}$ a basis of $\operatorname{Im} f$. If $f\left(s_{j}\right)=\sum_{i} f_{i j} \mathbf{v}_{i}$, then $f=\sum_{i, j} f_{i j} \widetilde{\varphi}_{j} \otimes \mathbf{v}_{i}$.

We define a trace on $\mathscr{L}_{0}(V)$ by:

$$
\varphi \in V^{*}, \mathbf{v} \in V, \quad \operatorname{Tr}(\varphi \otimes \mathbf{v})=\varphi(\mathbf{v}) .
$$

The usual properties of the trace are satisfied, as shown by the following lemma:

\subsubsection{Lemma.}

1. $\forall f \in \mathscr{L}_{0}(V), \forall g \in \mathscr{L}(V), \operatorname{Tr}(f \circ g)=\operatorname{Tr}(g \circ f)$.

2. Let $V_{1}$ and $V_{2}$ be t.v.s., $\operatorname{Tr}_{1}$ and $\operatorname{Tr}_{2}$ their respective traces, assuming that $u$ is an isomorphism from $V_{1}$ to $V_{2}$. One has:

$$
\forall f \in \mathscr{L}_{0}\left(V_{1}\right), \quad \operatorname{Tr}_{1}(f)=\operatorname{Tr}_{2}\left(u \circ f \circ u^{-1}\right) .
$$


Proof. To prove 1, we use (2.1.1) and restrict to $f=\varphi \otimes \mathbf{v}, \varphi \in V^{*}, \mathbf{v} \in V$. Then $f \circ g=(\varphi \circ g) \otimes \mathbf{v}$, and $g \circ f=\varphi \otimes[g(\mathbf{v})]$, so:

$$
\operatorname{Tr}(f \circ g)=\varphi[g(\mathbf{v})]=\operatorname{Tr}(g \circ f) .
$$

For 2, the same kind of arguments work.

2.2. For the purposes of deformation theory, we have to introduce formal maps, and formal trace. This is a straightforward generalization:

We define the $\mathbb{C}[[t]]$-algebra $\mathscr{L}(V)_{t}$ of formal linear maps by:

$$
\mathscr{L}(V)_{t}=\left\{\widetilde{f}=\sum_{n} f_{n} t^{n} ; f_{n} \in \mathscr{L}(V)\right\}
$$

with the product:

$$
\left(\sum_{n} f_{n} t^{n}\right) \circ\left(\sum_{n} g_{n} t^{n}\right)=\sum_{n}\left(\sum_{i=0}^{n} f_{i} \circ g_{n-i}\right) t^{n},
$$

and the ideal $\mathscr{L}_{0}(V)_{t}$ of formal finite rank maps by:

$$
\mathscr{L}_{0}(V)_{t}=\left\{\widetilde{f}=\sum_{n} f_{n} t^{n} ; f_{n} \in \mathscr{L}_{0}(V)\right\} .
$$

The formal trace $\operatorname{Tr}(\tilde{f})=\sum_{n} \operatorname{Tr}\left(f_{n}\right) t^{n} \in \mathbb{C}[[t]]$ is $\mathbb{C}[[t]]$-linear and satisfies:

$$
\operatorname{Tr}(\tilde{f} \circ \widetilde{g})=\operatorname{Tr}(\tilde{g} \circ \tilde{f}), \quad \forall \tilde{f} \in \mathscr{L}_{0}(V)_{t}, \forall \widetilde{g} \in \mathscr{L}(V)_{t}
$$

2.3. Let $G$ be a connected Lie group and $\pi$ a $C^{\infty}$ representation of $G$ in a t.v.s. $V$. Given $f \in \mathscr{L}_{0}(V)$, we define the coefficient $c_{f}^{\pi}$ by:

$$
c_{f}^{\pi}(x)=\operatorname{Tr}(f \circ[\pi(x)]), \quad x \in G .
$$

Using (2.1.1), it is clear that the space of coefficients $\operatorname{Coeff}(\pi)$ is a subspace of $C^{\infty}(G)$. Generalized coefficients are obtained when using formal finite rank maps.

Let $\boldsymbol{L}$ and $\boldsymbol{R}$ be the left and right regular representations of $G$ on $C^{\infty}(G)$. Both are $C^{\infty}$ representations. By (A.5), $\check{\pi}$ is a $C^{\infty}$ representation of $G$ on $V^{*}$, and one has, if $\varphi \in V^{*}, \mathbf{v} \in V$ :

$$
\left\{\begin{array}{l}
\boldsymbol{L}(x) c_{\varphi \otimes \mathbf{v}}^{\pi}=c_{[\check{\pi}(x) \varphi] \otimes \mathbf{v}}^{\pi}, \\
\boldsymbol{R}(x) c_{\varphi \otimes \mathbf{v}}^{\pi}=c_{\varphi \otimes[\pi(x) \mathbf{v}]}^{\pi} .
\end{array}\right.
$$

It results that $\operatorname{Coeff}(\pi)$ is stable under the left and right regular representations.

\subsubsection{Proposition.}

1. Assume that $W$ is a closed stable subspace of $V$ and let $\pi_{W}$ be the restriction of $\pi$ to $W$. Then $\operatorname{Coeff}\left(\pi_{W}\right) \subset \operatorname{Coeff}(\pi)$.

2. We suppose that $V$ is a Fréchet space and keep the assumptions of 1; let $\pi^{\dagger}$ be the $C^{\infty}$ quotient representation on $V / W$. Then $\operatorname{Coeff}\left(\pi^{\dagger}\right) \subset \operatorname{Coeff}(\pi)$.

3. If $\pi$ and $\pi^{\prime}$ are two equivalent $C^{\infty}$ representations of $G$, then $\operatorname{Coeff}(\pi)=$ $\operatorname{Coeff}\left(\pi^{\prime}\right)$. 
Proof. 1 is a trivial consequence of the Hahn-Banach Theorem. For 2, denote by $\mu: V \rightarrow V / W$ the canonical map, given $\varphi \in(V / W)^{*}$ and $\mathbf{v} \in V$, one has $c_{\varphi \otimes[\mu(\mathbf{v})]}^{\pi \dagger}=$ $c_{(\varphi \circ \mu) \otimes \mathbf{v}}^{\pi}$, then 2 follows from (2.1.1). Finally, 3 is an immediate consequence of (2.1.2.2).

Let us note that the restriction in 2 is essential because, for a general t.v.s. $V$, $V / W$ need not be a t.v.s., but is not really troublesome since we are especially interested in the case when $\pi$ is the restriction to the space of $C^{\infty}$ vectors of a continuous Banach representation of $G$, in which case $V$ is Fréchet space.

2.3.2. Proposition. Let $\pi$ and $\pi^{\prime}$ be two $C^{\infty}$ representations of $G$ on $V$ and $V^{\prime}$ respectively, $f \in \mathscr{L}_{0}(V), f^{\prime} \in \mathscr{L}_{0}\left(V^{\prime}\right)$. Then $c_{f}^{\pi} \times c_{f^{\prime}}^{\pi^{\prime}}=c_{f \otimes f^{\prime}}^{\pi \otimes}$.

Proof. By (A.5), $\pi \otimes \pi^{\prime}$ is a $C^{\infty}$ representation of $G$, by (2.1.1), $f \otimes f^{\prime} \in$ $\mathscr{L}_{0}\left(V \widehat{\otimes} V^{\prime}\right)$, and we can restrict to $f=\varphi \otimes \mathbf{v}, f^{\prime}=\varphi^{\prime} \otimes \mathbf{v}^{\prime}, \varphi \in V^{*}, \varphi^{\prime} \in V^{\prime *}$, $\mathbf{v} \in V, \mathbf{v}^{\prime} \in V^{\prime}$, in which case the formula is trivial.

2.4. Given a $C^{\infty}$ representation $\pi$ of $G$ on a t.v.s. $V$, we define its conjugate $\bar{\pi}$ as follows: first, we define a new t.v.s. structure, say $\bar{V}$, on $V$ by $\lambda \cdot \mathbf{v}=\bar{\lambda} \mathbf{v}, \lambda \in \mathbb{C}$, $\mathbf{v} \in V$; then we set $\bar{\pi}(x) \mathbf{v}=\pi(x) \mathbf{v}, x \in G, \mathbf{v} \in V$, and get a $C^{\infty}$ representation $\bar{\pi}$ of $G$ on $\bar{V}$. One has to take care that $\bar{\pi}$ is generally not equivalent to $\pi$, since $\operatorname{Id}_{\mathrm{V}}$ is antilinear from $V$ to $\bar{V}$. Now, there is an antilinear isomorphism between $V^{*}$ and $\bar{V}^{*}$ defined by $\bar{\varphi}(\mathbf{v})=\overline{\varphi(\mathbf{v})}, \varphi \in V^{*}$. So, coefficients of $\bar{\pi}$ are conjugate of coefficients of $\pi$, i.e. $\operatorname{Coeff}(\bar{\pi})=\overline{\operatorname{Coeff}(\pi)}$.

We say that $\pi$ is self-conjugate if $\bar{\pi} \simeq \pi$; obviously, $\pi \oplus \bar{\pi}$ is always selfconjugate.

2.5. Any $C^{\infty}$ representation $\pi$ of $G$ in a t.v.s. $V$ extends to a continuous representation of $A(G)$ (A.1), that we still denote $\pi$; on the other hand, for any coefficient $c_{f}^{\pi}$ of $\pi$, since $c_{f}^{\pi}$ belongs to $C^{\infty}(G)=A(G)^{*}$, it is a continuous form on $A(G)$. Now formula:

$$
\left\langle c_{f}^{\pi} \mid a\right\rangle=\operatorname{Tr}(f \circ[\pi(a)])
$$

is valid if $a \in G$, so, by the density of $\operatorname{Span}(G)$ in $A(G)$, it holds if $a \in A(G)$.

2.6. Let now $\pi$ be a representation of $G$ in a Banach space $V$. There are several possible choices for what could be called " $C^{\infty}$ coefficients of $\pi$," the simplest one being the space of coefficients of $\left.\pi\right|_{V_{\infty}}=\pi_{\infty}$. But this last space contains functions which are not continuous coefficients of $\pi$ in the usual sense. So we define the space of $C^{\infty}$ coefficients of $\pi$ as follows:

We consider $V^{*} \otimes V_{\infty}$ and we define $\operatorname{Coeff}_{\infty}(\pi)$ to be the subspace of $\operatorname{Coeff}\left(\pi_{\infty}\right)$ generated by $c_{f}^{\pi_{\infty}}, f \in V^{*} \otimes V_{\infty}$. The space $\operatorname{Coeff}_{\infty}(\pi)$ is still stable under the left and right regular representations of $G$ on $C^{\infty}(G)$.

Similarly, we define the space of analytic coefficients of $\pi$ to be the subspace $\operatorname{Coeff}_{\omega}(\pi)$ of $\operatorname{Coeff}\left(\pi_{\infty}\right)$ generated by $C_{f}^{\pi_{\infty}}, f \in V^{*} \otimes V_{\omega}$. As for $\operatorname{Coeff}_{\infty}(\pi)$, one has stability of $\operatorname{Coeff}_{\omega}(\pi)$ under the left and right regular representations. Moreover, $\operatorname{Coeff} \omega(\pi) \subset C^{\omega}(G)$, so analytic coefficients are completely specified by their restriction to $\mathscr{U}(\mathfrak{g})$, since an analytic function is specificed by its Taylor series. Finally, any subalgebra of $C^{\infty}(G)$ generated by analytic coefficients is a domain. 
One can define the conjugate $\bar{\pi}$ of $\pi$ as in (2.4). $\pi$ and $\bar{\pi}$ have exactly the same $C^{\infty}$ or $C^{\omega}$ vectors, and $\bar{V}_{\infty}=\overline{V_{\infty}}$ as t.v.s.. Moreover, since $V^{*}$ and $(\bar{V})^{*}$ are anti-isomorphic by $\bar{\varphi}(\mathbf{v})=\overline{\varphi(\mathbf{v})}, \varphi \in V^{*}, \mathbf{v} \in V$, one has:

$$
\operatorname{Coeff}_{\infty}(\bar{\pi})=\overline{\operatorname{Coeff}_{\infty}(\pi)} \text { and } \operatorname{Coeff}_{\omega}(\bar{\pi})=\overline{\operatorname{Coeff}_{\omega}(\pi)}
$$

Note that, if $\pi$ is a unitary representation, then $\bar{\pi} \simeq \check{\pi}$, the contragredient of $\pi$.

2.7. Given a family $\Pi$ of representations of $G$, we define subalgebras of $C^{\infty}(G)$ as follows:

- when any element of $\Pi$ is a $C^{\infty}$ representation, then $\mathbb{C}[\Pi]$ is the subalgebra of $C^{\infty}(G)$ generated by all coefficients of all elements of $\Pi$;

- when $\Pi$ is a family of Banach representations, then $\mathbb{C}\left[\Pi_{\infty}\right]$ is the subalgebra of $C^{\infty}(G)$ generated by $C^{\infty}$ coefficients of all elements of $\Pi$ and $\mathbb{C}\left[\Pi_{\omega}\right]$ is the subalgebra of $C^{\omega}(G)$ generated by $C^{\omega}$ coefficients of all elements of $\Pi$.

In any case, those algebras are all stable under the left and right regular representations. If $\Pi$ is assumed stable by conjugation, then the same property holds for the associated subalgebras.

Clearly, the most interesting case is the algebra $\mathbb{C}\left[\Pi_{\omega}\right]$, because this algebra is a domain (2.6). Note that the family $\Pi_{f}$ of finite-dimensional representations is in both cases but there is no ambiguity because any vector is analytic and:

$$
\mathbb{C}\left[\Pi_{f}\right]=\mathbb{C}\left[\left(\Pi_{f}\right)_{\infty}\right]=\mathbb{C}\left[\left(\Pi_{f}\right)_{\omega}\right]
$$

2.8. We shall now use the $C^{\infty}$ Stone-Weierstrass Theorem of Appendix B to obtain some density results on the algebras defined in (2.7).

2.8.1. Proposition. Let $\Pi$ be the family of all unitary irreducible representations of $G$; then $\mathbb{C}\left[\Pi_{\omega}\right]$ is dense in $C^{\infty}(G)$.

Proof. The Gel'fand-Raikov Theorem asserts that $G$ has sufficiently many unitary irreducible representations, so $\mathbb{C}\left[\Pi_{\omega}\right]$ satisfies the assumptions of the $C^{\infty}$ StoneWeierstrass Theorem of Appendix B.

2.8.2. Proposition. If $G$ is linear, then $\mathbb{C}\left[\Pi_{f}\right]$ is dense in $C^{\infty}(G)$.

Proof. Let $\pi$ be any f.d. faithful representation of $G$, then $\mathbb{C}[\pi, \bar{\pi}]$ satisfies the assumptions of the $C^{\infty}$ Stone-Weierstrass Theorem of Appendix B.

\subsubsection{Remarks.}

1. When $G$ is compact, $G$ is linear and, if $\pi$ is any f.d. faithful and self-conjugate representation of $G$, then $\mathbb{C}[\pi]=\mathbb{C}\left[\Pi_{f}\right]([5$ or 7$])$.

2. When $G$ is linear and $\pi$ is any f.d. faithful and self-conjugate representation, then $\mathbb{C}[\pi]$ is dense in $C^{\infty}(G)$ by the proof of (2.8.2) but it can happen that $\mathbb{C}[\pi] \neq \mathbb{C}\left[\Pi_{f}\right]$ (see an example in $[5]$ ).

3. If $G$ is semisimple, then $G$ is linear if and only if $\mathbb{C}\left[\Pi_{f}\right]$ is dense in $C^{\infty}(G)$ ([5]); moreover, for any semisimple linear group, it is always possible to find a f.d. representation such that $\mathbb{C}[\pi]=\mathbb{C}\left[\Pi_{f}\right]([5])$. 


\section{Admissible Coefficients}

In this section, we assume once and for all that $G$ is a connected semisimple Lie group with finite center. We fix a maximal compact subgroup $K$ and denote by $\mathrm{t}_{0}$ the Lie algebra and by $t$ the complexification of $t_{0}$. We make a free use of the terminology of Appendix A.

3.1. Let $\pi$ an admissible representation of $G$ in a Banach space $V, V_{K}$ its space of $K$-finite vectors, which is an $\mathscr{U}(\mathfrak{g})$-module, and $V_{K}^{\circledast}$ the dual Harish-Chandra module.

We consider the space $V_{K}^{\circledast} \otimes V_{K}$, and the corresponding finite rank linear mappings defined by: $(\varphi \otimes \mathbf{v})(\mathbf{w})=\varphi(\mathbf{w}) \mathbf{v}, \varphi \in V_{K}^{\circledast}, \mathbf{v}, \mathbf{w} \in V_{K}$.

As noted in [21], from the existence of continuous projections on each isotypical component of $K$, it results that each element $f \in V_{K}^{\circledast} \otimes V_{K}$ can be extended to a (finite rank) continuous linear mapping from $V$ into $V$ (or $V_{\infty}$ into $V_{\infty}$ ), so that $f \in V^{*} \otimes V_{\omega}$. Therefore, using (2.6), $f$ defines an analytic coefficient $c_{f}^{\pi}$ by the formula:

$$
c_{f}^{\pi}(x)=\operatorname{Tr}(f \circ[\pi(x)]), \quad x \in G .
$$

In the sequel, coefficients of this type will be called $K$-finite, or admissible coefficients of $\pi$; the space of admissible coefficients will be denoted $\operatorname{Coeff}_{K}(\pi)$. Since $\operatorname{Coeff}_{K}(\pi) \subset C^{\omega}(G)$, an admissible coefficient is completely specified by its restriction on $\mathscr{U}(\mathfrak{g})$. It has to be noted that $V_{K}$ is generally not stable under $\pi$, so $\operatorname{Coeff}_{K}(\pi)$ is generally not stable under the right or left regular representation of $G$; nevertheless, $\operatorname{Coeff}_{K}(\pi)$ is stable under the restriction to $K$ of the right and left regular representations, and also under the left and right regular as representations of $\mathscr{U}(\mathfrak{g})$, because $V_{K}$ is stable under $\left.\pi\right|_{K}$, and also under $\left.\pi\right|_{\mathscr{U}(\mathrm{g})}$, moreover, $V_{K}^{\circledast}$ is stable under $\left.\check{\pi}\right|_{K}$, and also under $\left.\check{\pi}\right|_{\mathscr{U}(\mathrm{g})}$ (see (2.5) or Appendix A for the notations).

Generalized admissible coefficients are defined by formal series $\sum_{n} c_{f_{n}}^{\pi} t^{n}$, with $c_{f_{n}}^{\pi}$ admissible coefficients of $\pi, \forall n$.

3.2. We want to state formula (2.3.2) in the case of admissible coefficients; obviously, the tensor product of two admissible representations is generally not admissible as a representation of $G$; fortunately, it is admissible as a representation of $G \times G((\mathrm{~A} .10))$, so we can state:

3.2.1. Proposition. Let $\pi$ and $\pi^{\prime}$ be admissible Banach representations of $G, c_{f}^{\pi}$ and $c_{f^{\prime}}^{\pi^{\prime}}$ be admissible coefficients. Then $c_{f \otimes f^{\prime}}^{\pi \otimes \pi^{\prime}}$ is an admissible coefficient of the representation $\pi \otimes \pi^{\prime}$ of $G \times G$, and one has:

$$
c_{f}^{\pi} \times c_{f^{\prime}}^{\pi^{\prime}}=\left.c_{f \otimes f^{\prime}}^{\pi \otimes \pi^{\prime}}\right|_{G} .
$$

3.2.2. Corollary 1. The space of restrictions to $G$ of admissible coefficients of the representation $\pi \otimes \pi^{\prime}$ of $G \times G$, is exactly the space generated in $C^{\omega}(G)$ by products of admissible coefficients of $\pi$ and $\pi^{\prime}$.

Before we give a proof, let us make some remarks.

1. The corollary is easily generalized to $n$ tensor products, so, one obtains:

3.2.3. Corollary 2. The subalgebra of $C^{\omega}(G)$ generated by admissible coefficients of $\pi$ and $\pi^{\prime}$ is exactly the subspace generated by restrictions to $G$ of admissible 
coefficients of representations $\pi_{1} \otimes \cdots \otimes \pi_{n}, \pi_{i}=\pi$ or $\pi^{\prime}, n \in \mathbb{N}^{*}$, of $G^{n}$, and the constant function 1.

2. The results work as well for Hilbert representations and the Hilbertian tensor product.

Proof of the Proposition. We can restrict to $f=\varphi \otimes \mathbf{v}, f^{\prime}=\varphi^{\prime} \otimes \mathbf{v}^{\prime}, \varphi \in V_{K}^{\circledast}$, $\varphi^{\prime} \in\left(V^{\prime}\right)_{K}^{\circledast}, \mathbf{v} \in V_{K}, \mathbf{v}^{\prime} \in V_{K}^{\prime}$. Then $f \otimes f^{\prime}=\left(\varphi \otimes \varphi^{\prime}\right) \otimes\left(\mathbf{v} \otimes \mathbf{v}^{\prime}\right)$, one has $\varphi \otimes \varphi^{\prime} \in$ $\left(V \widehat{\otimes} V^{\prime}\right)_{K \times K}^{\circledast}, \mathbf{v} \otimes \mathbf{v}^{\prime} \in\left(V \widehat{\otimes} V^{\prime}\right)_{K \times K}$ by (A.10), so $c_{f \otimes f^{\prime}}^{\pi \otimes \pi^{\prime}}$ is an admissible coefficient of the representation $\pi \otimes \pi^{\prime}$ of $G \times G$. Formula (3.A) is trivial.

3.3. Given a set $\Pi$ of admissible Banach representations of $G$, we denote by $\mathbb{C}_{K}[\Pi]$ the subalgebra of $C^{\infty}(G)$ generated by admissible coefficients of all elements in $\Pi$. Since $\mathbb{C}_{K}[\Pi]$ is contained in $C^{\omega}(G)$, the algebra $\mathbb{C}_{K}[\Pi]$ is a domain. Following the usual terminology, we say that $\Pi$ contains sufficiently many representations if, for any $x \in G \backslash\{e\}$, one can find $\pi \in \Pi$ such that $\pi(x) \neq$ Id.

3.3.1. Proposition. If $\Pi$ is stable by conjugation and contains sufficiently many representations, then $\mathbb{C}_{K}[\Pi]$ is dense in $C^{\infty}(G)$.

Let us note that, when $\Pi$ contains only unitary representations, stability of $\Pi$ under conjugation is equivalent to stability of $\Pi$ under contragredient operation.

Proof. $\mathbb{C}_{K}[\Pi]$ satisfies all assumptions of the $C^{\infty}$ Stone-Weierstrass Theorem (use (A.4) for assumption 2), except one: stability under the right regular representation (see (3.1)); we introduce the closure $\overline{\mathbb{C}_{K}[\Pi]}$ in $C^{\infty}(G)$.

Let $\pi \in \Pi, V_{\infty}$ be the space of $C^{\infty}$ vectors of $\pi$ and $\pi_{\infty}$ the restriction of $\pi$ to $V_{\infty}$. Given $\mathbf{v} \in V_{\infty}$, we define $f_{\mathbf{v}} \in C^{\infty}\left(G, V_{\infty}\right)$ by $f_{\mathbf{v}}(x)=\pi_{\infty}(x) \mathbf{v}$. The map $\mathbf{v} \mapsto f_{\mathbf{v}}$ is an isomorphism from $V_{\infty}$ onto a closed subspace of $C^{\infty}\left(G, V_{\infty}\right)$ ([25]). Let $\boldsymbol{R}$ be the right regular representation of $G$ on $C^{\infty}(G)$, and $c_{\varphi \otimes v}^{\pi}, \varphi \in V_{K}^{\circledast}$, $\mathbf{v} \in V_{K}$, an admissible coefficient of $\pi$. Then $\boldsymbol{R}(x) c_{\varphi \otimes \mathbf{v}}^{\pi}=\varphi \circ f_{\pi_{\infty}(x) \mathbf{v}}$. Since $V_{K}$ is dense in $V_{\infty}$, there is a sequence $\mathbf{v}_{n} \in V_{K}$ such that $\pi_{\infty}(x) \mathbf{v}=\lim _{n} \mathbf{v}_{n}$ in $V_{\infty}$; therefore $f_{\pi_{\infty}(x) \mathbf{v}}=\lim _{n} f_{\mathbf{v}_{n}}$ in $C^{\infty}\left(G, V_{\infty}\right)$, and since $\varphi \in V_{\infty}^{*}$, one has $\lim _{n}\left(\varphi \circ f_{\mathbf{v}_{n}}\right)=$ $\varphi \circ f_{\pi_{\infty}(x) \mathbf{v}}$ in $C^{\infty}(G)$, i.e. $\lim _{n} c_{\varphi \otimes \mathbf{v}_{n}}^{\pi}=\boldsymbol{R}(x) c_{\varphi \otimes \mathbf{v}}^{\pi}$. As consequence, $\overline{\mathbb{C}_{K}[\Pi]}$ is stable under the right regular representation, so it satisfies all assumptions of the $C^{\infty}$ Stone-Weierstrass Theorem.

3.4. Using (3.3), we shall now propose some refinements of (2.8.1). Our goal is to find finite sets $\Pi$ of irreducible Banach representations of $G$ such that $\mathbb{C}_{K}[\Pi]$ is dense in $C^{\infty}(G)$. For instance, if $G$ is linear, it is possible to find such a set containing only f.d. representations $((2.8 .2))$. If we drop the assumption that $G$ is linear, then, by (2.8.3.3), we have to use infinite-dimensional representations. Let us now show that the classical construction of the principal series will give an answer to the question.

3.4.1. Proposition. Let $H$ be a closed normal subgroup of $G$. Then $H \cap K=\{e\}$ implies $H=\{e\}$.

3.4.2. Corollary. Let $\pi$ be a representation of $G$. Then $\pi$ is faithful if and only if $\left.\pi\right|_{K}$ is faithful.

Proof. Let $\mathfrak{h}_{0}$ be the Lie algebra of $H$, and $H_{e}$ be the connected component of $\{e\}$ in $H$. Then $H_{e}$ is a closed normal semisimple subgroup of $G$, with Lie algebra $\mathfrak{h}_{0}$. 
Since $\mathfrak{h}_{0}$ is an ideal in $\mathfrak{g}_{0}$, we have $\mathfrak{g}_{0}=\mathfrak{h}_{0} \oplus \mathfrak{i}$, with $\mathfrak{i}$ an ideal. Since $\left[\mathfrak{h}_{0}, \mathfrak{i}\right]=\{0\}$, and $H_{e}$ is connected, $\left.\mathrm{Ad}\right|_{H_{e}}$ acts trivially on $\mathfrak{i}$, and, on $\mathfrak{h}_{0}$, it acts as the Adjoint representation of $H_{e}$. So, $\operatorname{Ker}\left(\left.\operatorname{Ad}\right|_{H_{e}}\right)=Z\left(H_{e}\right)$, the center of $H_{e}$, but since $\operatorname{Ker}\left(\left.\operatorname{Ad}\right|_{H_{e}}\right)=H_{e} \cap Z(G)$, where $Z(G)$ is the center of $G$, which is finite, we conclude that $Z\left(H_{e}\right)$ is finite.

Let $K^{\prime}$ be a maximal compact subgroup of $H_{e}$. Since $H_{e}$ is connected, semisimple and has finite center, one has: $K^{\prime}=\{e\} \Leftrightarrow H_{e}=\{e\}$. There exists $x \in G$ such that $x K^{\prime} x^{-1} \subset K$; since $H$ is normal, we can assume from the beginning that $K^{\prime} \subset K$. It results that: $H \cap K=\{e\} \Rightarrow H_{e}=\{e\}$. Now, if $H_{e}=\{e\}, H$ is a discrete closed normal subgroup, but $G$ is connected, so $H \subset Z(G)$. Now, it is well-known that $Z(G) \subset K$, so $H=\{e\}$.

For the corollary, we apply (3.4.1) to $H=\operatorname{Ker} \pi$.

Let now $G=K A N$ be an Iwasawa decomposition of $G, M$ the centralizer of $A$ in $K$, and $P=M A N$ the corresponding minimal parabolic subgroup of $G$. The unitary principal $P$-series of $G$ is obtained when inducing unitary representations of $P$ of type $\sigma(\operatorname{man})=\xi(a) L(m), \xi \in \widehat{A}, L \in \widehat{M}$. By the Frobenius reciprocity Theorem, given $\delta \in \widehat{K}$, then $\delta$ is contained in $\operatorname{Ind}_{P \uparrow G} \sigma$ as many times as $L$ is contained in $\left.\delta\right|_{M}$. So, using (3.4.2), we can easily construct faithful representations of $G$ :

3.4.3. Proposition. Let $\delta$ be a faithful unitary representation of $K$, and $\delta=$ $\delta_{1} \oplus \cdots \oplus \delta_{r}, \delta_{i} \in \widehat{K}$, its reduction into irreducible; for $i=1, \ldots r$, let $L_{i} \in \widehat{M}$ be an irreducible component of $\left.\delta_{i}\right|_{M}, \xi_{i} \in \widehat{A}, \quad \sigma_{i}=\xi_{i} \cdot L_{i}, v_{i}=\operatorname{Ind}_{P \uparrow G} \sigma_{i}$, and $v=\bigoplus_{i=1}^{r} v_{i}$. Then $v$ is a faithful unitary representation of $G$.

Proof. By the construction of $v$, one has $\left.\delta \subset v\right|_{K}$, so $v$ is faithful by (3.4.2). For the exitence of $\delta$, see e.g. Chevalley [7].

3.4.4. Corollary. There exist unitary irreducible representations $\pi_{1}, \ldots, \pi_{n}$ of $G$ such that $\mathbb{C}_{K}\left[\pi_{1}, \ldots, \pi_{n}\right]$ is dense in $C^{\infty}(G)$.

Proof. Any unitary principal $P$-series representation is known to be reducible into a finite sum of irreducible ([25]), so, with the notations of (3.4.3), $v$ is finitely reducible, and so does $\check{v} ; \pi_{1}, \ldots, \pi_{n}$ are obtained as the distinct irreducible components of the reductions of $\pi=v \oplus \check{v}$ and (3.4.4) follows from (3.3.1).

\subsubsection{Remarks.}

1. Since the $\xi_{i} \in \widehat{A}$ are arbitrary in (3.4.3), one has some freedom in the choice of the representations $\pi_{1}, \ldots, \pi_{n}$ of (3.4.4), for instance, for their infinitesimal characters.

2. For any compact group $K$, the statement of (3.4.4) is true (see (2.8.3.1)). Proposition (3.4.3) and Corollary (3.4.4) can be seen as a direct consequence, using the maximal compact $K$ of $G$, and the induction process of principal $P$-series.

3. In the case of $G=\mathbf{S U}(1,1)$, by a good choice, one can take $n=1$ in (3.4.4). This is not true in general (even for a compact $G$ ).

3.5. In this subsection, we assume that $\operatorname{rank} G=\operatorname{rank} K$. This is exactly the case when $G$ has discrete series representations: we shall deal with a restricted case, the so-called "holomorphic discrete series." Indeed, our assumption $\operatorname{rank} G=\operatorname{rank} K$ is clearly not enough for the existence of such representations. Moreover, we are mainly interested in the algebraic structure of our representations, rather than their 
square-integrability. So let us assume that we consider a group $G$ with the following property: Having fixed a compact Cartan subalgebra $\mathfrak{h}$, there exists a Borel subalgebra $\mathfrak{b}$ relative to $\mathfrak{h}$, and a (nontrivial) admissible unitary representation such that the associated Harish-Chandra module is generated by an extreme weight vector of $\mathfrak{h}$ (i.e. a vector $\mathbf{v}$ such that $\mathbb{C} \mathbf{v}$ is $\mathfrak{b}$-stable).

We shall call such a representation a $\mathfrak{b}$-extreme unitary representation of $G$. We shall not discuss the conditions on $G$ in order that nontrivial b-extreme unitary representations do exist, we refer to Harish-Chandra [16] or Varadarajan [24]. Let us note that though this type of representations was initially introduced by HarishChandra in order to construct discrete series representations (and he gave conditions to ensure square-integrability), it can happen that they are not square-integrable (e.g. the mock discrete series of $\mathbf{S U}(1,1))$. Finally, since $\mathfrak{b}$-extreme $\mathscr{U}(\mathfrak{g})$-modules are indecomposable, a unitary $\mathfrak{b}$-extreme representation of $G$ is actually irreducible.

3.5.1 Lemma. Let $\pi$ be a unitary admissible representation of $G$ in $V$. Then $V_{K}$ is a semisimple $\mathscr{U}(\mathfrak{g})$-module, and $\pi$ reduces as a direct orthogonal sum of unitary irreducible representations.

Remark. Lemma (3.5.1) is true for any semisimple group $G$ with finite center.

Proof. Given a $\mathscr{U}(\mathfrak{g})$-submodule $W$ of $V_{K}$, since $\pi(x)\left(x \in \mathfrak{g}_{0}\right)$ is antisymmetric, the subspace $W^{\perp}=\left\{\mathbf{v} \in V_{K} / \mathbf{v} \perp W\right\}$ is also a submodule. One has $W=\bigoplus_{\delta \in \widehat{K}}\left(W \cap V_{\delta}\right)$, the $V_{\delta}$ are mutually orthogonal and finite-dimensional, so $V_{K}=W \oplus W^{\perp}$, which proves that $V_{K}$ is a semisimple $\mathscr{U}(\mathfrak{g})$-module. By Zorn's lemma, we can reduce $V_{K}$ into a direct sum of mutually orthogonal simple $\mathscr{U}(\mathfrak{g})$-submodules, i.e. $V_{K}=$ $\bigoplus_{i \in I} V_{i}$. By (A.6), $\bar{V}_{i}$ is $G$-stable, and the restriction of $\pi$ to $\bar{V}_{i}$ is a unitary irreducible representation; moreover, $V=\bigoplus_{i \in I} \bar{V}_{i}$.

\subsubsection{Lemma.}

1. Let $V$ be an admissible $\mathscr{U}(\mathfrak{g})$-module; assume that $V=\bigoplus_{i \in I} W_{i}$, where $W_{i}$ are submodules. Then $V_{\mathrm{t}}^{\circledast}=\bigoplus_{i \in I} W_{i}^{\circledast}$.

2. Let $\pi$ an admissible representation of $G$ in a Banach space $V$; assume that $V_{K}=\bigoplus_{i \in I} W_{i}$, where $W_{i}$ are $\mathscr{U}(\mathfrak{g})$-submodules. Then $\bar{W}_{i}$ is $G$-stable, the restriction $\pi_{i}$ of $\pi$ to $\bar{W}_{i}$ is admissible, and $\operatorname{Coeff}_{K}(\pi)=\sum_{i \in I} \operatorname{Coeff}\left(\pi_{i}\right)$ (non-direct sum).

Remark. Lemma (3.5.2) is true for any semisimple group $G$ with finite center.

Proof.

1. First, one has $\left(W_{i}\right)_{\delta}=W_{i} \cap V_{\delta}, \delta \in \widehat{\mathrm{t}}$, so that each $W_{i}$ is admissible. If $P_{i}$ is the projection on $W_{i}$, with respect to the reduction $V=\bigoplus_{j \in I} W_{j}$, then $P_{i}\left(V_{\delta}\right) \subset$ $\left(W_{i}\right)_{\delta}$, so $V_{\delta}=\bigoplus_{i \in I}\left(W_{i}\right)_{\delta}$. If fact, the last sum is finite, since $\operatorname{dim} V_{\delta}<\infty$, so $V_{\delta}^{*}=$ $\bigoplus_{i \in I}\left(W_{i}\right)_{\delta}^{*}$, and then

$$
V^{\circledast}=\bigoplus_{\delta \in \widehat{\mathfrak{t}}} V_{\delta}^{*}=\bigoplus_{\substack{\delta \in \hat{t} \\ i \in I}}\left(W_{i}\right)_{\delta}^{*}=\bigoplus_{i \in I} W_{i}^{\circledast} .
$$

2. By (A.6), $\bar{W}_{i}$ is a subrepresentation, and $\left(\bar{W}_{i}\right)_{K}=W_{i}$, so $\overline{W_{i}}$ is admissible. Using $1, K$-finite coefficients of $\pi_{i}$ are $K$-finite coefficients of $\pi$, and any $K$-finite coefficient of $\pi$ is a (finite) sum of $K$-finite coefficients of $\pi_{i}, i \in I$.

3.5.3. Remark. We can apply (3.5.2) to the case of (3.5.1): if $\pi$ is a unitary admissible representation of $G$, then $\pi$ reduces into a direct sum of unitary 
irreducible $\pi_{i}, i \in I$, and any $K$-finite coefficient of $\pi$ is a (finite) sum of $K$-finite coefficients of $\pi_{i}, i \in I$.

3.5.4. Proposition. Let $\pi$ and $\pi^{\prime}$ be two b-extreme unitary representations of $G$, respectively in $V$ and $V^{\prime}$, and $\pi \otimes \pi^{\prime}$ their Hilbertian tensor product. Then $\pi \otimes \pi^{\prime}$ is unitary admissible, $\left(V \otimes_{2} V^{\prime}\right)_{K}=V_{K} \otimes V_{K}^{\prime}$, and $\pi \otimes \pi^{\prime}$ reduces into a direct sum of mutually orthogonal $\mathfrak{b}$-extreme unitary representations of $G$.

Proof. Let $\delta_{1}, \delta_{2} \in \widehat{K}$. Then $V_{\delta_{1}} \otimes V_{\delta_{2}}$ is stable under $\left.\pi \otimes \pi^{\prime}\right|_{K}$, so also stable under the projection $P_{\delta}$ onto $\left(V \otimes_{2} V^{\prime}\right)_{\delta}$, for any $\delta \in \widehat{K}$. It results that $V_{K} \otimes V_{K}^{\prime}$ is stable under $P_{\delta}$. Since $V_{K}$ and $V_{K}^{\prime}$ are $\mathfrak{h}$-diagonal modules, $V_{K} \otimes V_{K}^{\prime}$ is also h-diagonal.

Let $\lambda$ and $\lambda^{\prime}$ be the extreme weights of $V_{K}$ and $V_{K}^{\prime},\left\{\alpha_{1}, \ldots, \alpha_{n}\right\}$ a simple system of positive roots relative to $\mathfrak{b}$. Then any weight of $V_{K}$ (resp. $V_{K}^{\prime}$ ) is of the form: $\lambda-\sum_{i} n_{i} \alpha_{i}, n_{i} \in \mathbb{N}$ (resp. $\lambda^{\prime}-\sum_{i} n_{i}^{\prime} \alpha_{i}, n_{i}^{\prime} \in \mathbb{N}$ ), and the corresponding weight space is finite dimensional, so it results that any weight of $V_{K} \otimes V_{K}^{\prime}$ is of the form: $\lambda+\lambda^{\prime}-\sum_{i} m_{i} \alpha_{i}, m_{i} \in \mathbb{N}$, and that the corresponding weight space is finitedimensional. $V_{K} \otimes V_{K}^{\prime}$ is clearly $K$-finite, and, since its weight spaces are finitedimensional, it is an admissible $\mathscr{U}(\mathfrak{g})$-module.

Now, given $\mathbf{v} \in\left(V \otimes_{2} V^{\prime}\right)_{\delta}$, by the density of $V_{K} \otimes V_{K}^{\prime}$, we can find a sequence $\mathbf{v}_{n} \in V_{K} \otimes V_{K}^{\prime}$ such that $\mathbf{v}=\lim _{n} \mathbf{v}_{n}$. Then $\mathbf{v}=P_{\delta}(\mathbf{v})=\lim _{n} P_{\delta}\left(\mathbf{v}_{n}\right)$, but $P_{\delta}\left(\mathbf{v}_{n}\right) \in$ $\left(V_{K} \otimes V_{K}^{\prime}\right)_{\delta}$, which is finite-dimensional, so $\left(V \otimes_{2} V^{\prime}\right)_{\delta}=\left(V_{K} \otimes V_{K}^{\prime}\right)_{\delta}$, therefore $\left(V \otimes_{2} V^{\prime}\right)_{K}=V_{K} \otimes V_{K}^{\prime}$, and $\pi \otimes \pi^{\prime}$ is admissible. So (3.5.1) can be applied to reduce $\pi \otimes \pi^{\prime}$ into a direct sum of mutually orthogonal unitary irreducible $\pi_{i}$ acting on the stable subspaces $\bar{W}_{i}$ which come from the reduction $V_{K} \otimes V_{K}^{\prime}=\bigoplus_{i \in I} W_{i}$ of the $\mathscr{U}(\mathfrak{g})$-module $\left(V \otimes_{2} V^{\prime}\right)_{K}$ into mutually orthogonal simple submodules $W_{i}$. From the structure of the weight system of $V_{K} \otimes V_{K}^{\prime}$ described previously, it results that each $W_{i}$ is generated by an extreme weight vector, so each $\pi_{i}$ is a $\mathfrak{b}$-extreme unitary representation of $G$.

3.5.5. Proposition. Let $\Pi_{\mathfrak{b}}$ be the set of unitary $\mathfrak{b}$-extreme representations of $G$ (up to equivalence), including the trivial representation $\pi^{0}$. Then $\operatorname{Coeff}_{K}\left(\Pi_{\mathfrak{b}}\right)=$ $\bigoplus_{\pi \in \Pi_{\mathfrak{b}}} \operatorname{Coeff}_{K}(\pi)$ and $\operatorname{Coeff}_{K}\left(\Pi_{\mathfrak{b}}\right)$ is a subalgebra of $C^{\omega}(G)$. If $\Pi_{\mathfrak{b}}^{1}$ is the subset of integrable elements of $\Pi_{\mathfrak{b}}$, and $\Pi_{\mathfrak{b}}^{2}$ the subset of square-integrable elements of $\Pi_{\mathfrak{b}}$, then $\operatorname{Coeff}_{K}\left(\Pi_{\mathfrak{b}}^{1}\right) \subset \operatorname{Coeff}_{K}\left(\Pi_{\mathfrak{b}}^{2}\right)$ and $\operatorname{Coeff}_{K}\left(\Pi_{\mathfrak{b}}^{2}\right) \times \operatorname{Coeff}_{K}\left(\Pi_{\mathfrak{b}}^{2}\right) \subset$ $\operatorname{Coeff}_{K}\left(\Pi_{\mathfrak{b}}^{1}\right)$.

Remark. $\mathfrak{b}$-extreme $\mathscr{U}(\mathfrak{g})$-modules are completely determined by their extreme weight ([16]), so, due to the compactness of $K, \Pi_{\mathfrak{b}}$ is countable.

Proof. Given $\pi \in \Pi_{\mathfrak{b}}$, acting on $V$, Coeff ${ }_{K}(\pi)$ is linearly spanned by coefficients of type $c_{f}^{\pi}$, with $f=\varphi \otimes \mathbf{v}, \varphi \in V_{K}^{\circledast}$ and $\mathbf{v} \in V_{K}$. Let $\boldsymbol{R}$ be the right regular representation of $G$ on $C^{\infty}(G): \boldsymbol{R}$ is a $C^{\infty}$ representation of $G$ and one has:

$$
\boldsymbol{R}(X) c_{\varphi \otimes \mathbf{v}}^{\pi}=c_{\varphi \otimes[\pi(X) \mathbf{v}]}^{\pi}, \quad X \in \mathfrak{g}_{0},
$$

so the map $\mathbf{v} \in V_{K} \mapsto c_{\varphi \otimes \mathbf{v}}^{\pi}$, for fixed $\varphi$ in $V_{K}^{\circledast}$, is a $\mathscr{U}(\mathfrak{g})$-morphism from $V_{K}$ into $C^{\infty}(G)$. Since $V_{K}$ is a simple $\mathscr{U}(\mathfrak{g})$-module, if $\varphi$ is nonzero, the previous map is injective, so Coeff $K_{K}(\pi)$ is a semi-simple $\mathscr{U}(\mathfrak{g})$-module for the right regular representation, and it is isotypical of type $\pi$. It results that $\operatorname{Coeff}_{K}\left(\Pi_{\mathfrak{b}}\right)$ is also a semisimple $\mathscr{U}(\mathfrak{g})$-module, with isotypical component of type $\pi$ exactly $\operatorname{Coeff}_{K}(\pi)$, and this proves the first claim. 
Let now $\pi$ and $\pi^{\prime} \in \Pi_{\mathfrak{b}}$, we want to compute the product of two admissible coefficients by formula (3.A): $c_{f}^{\pi} \times c_{f^{\prime}}^{\pi^{\prime}}=\left.c_{f \otimes f^{\prime}}^{\pi \otimes \pi^{\prime}}\right|_{G}$. In this formula, $\pi \otimes \pi^{\prime}$ is the Hilbertian tensor product representation of $G \times G$. By (3.5.4), $\pi \otimes \pi^{\prime}$, as a representation of $G$, is admissible. One has: $\left(V \otimes_{2} V^{\prime}\right)_{K}=V_{K} \otimes V_{K}^{\prime}$, so:

and

$$
\left(V \otimes_{2} V^{\prime}\right)_{K}^{\circledast}=V_{K}^{\circledast} \otimes V_{K}^{\prime \circledast}
$$

$$
\left(V \otimes_{2} V^{\prime}\right)_{K}^{\circledast} \otimes\left(V \otimes_{2} V^{\prime}\right)_{K}=\left(V_{K}^{\circledast} \otimes V_{K}\right) \otimes\left(V_{K}^{\prime \circledast} \otimes V_{K}^{\prime}\right),
$$

so $\left.c_{f \otimes f^{\prime}}^{\pi \otimes \pi^{\prime}}\right|_{G}$ is an admissible coefficient of the representation $\pi \otimes \pi^{\prime}$ of $G$, and any admissible coefficient of that representation is a finite sum of such ones. Therefore, Coeff $_{K}\left(\pi \otimes \pi^{\prime}\right)$ is the subspace of $C^{\omega}(G)$ spanned by all products of admissible coefficients of $\pi$ and $\pi^{\prime}$.

On the other hand, applying (3.5.4), we reduce $\pi \otimes \pi^{\prime}=\bigoplus_{i \in I} \pi_{i}$ into b-extreme unitary irreducible representations of $G$, and (3.5.2) shows that $\operatorname{Coeff}_{K}\left(\pi \otimes \pi^{\prime}\right)=$

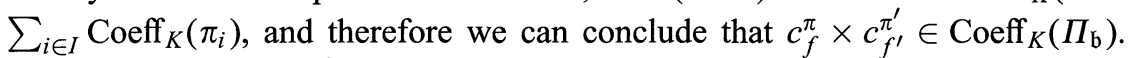

When $\pi$ and $\pi^{\prime}$ are square-integrable, Schwarz inequality shows that $c_{f}^{\pi} \times c_{f^{\prime}}^{\pi^{\prime}} \in \mathscr{L}^{1}(G)$, so $\operatorname{Coeff}_{K}\left(\pi \otimes \pi^{\prime}\right) \subset \mathscr{L}^{1}(G)$, and then $\operatorname{Coeff}_{K}\left(\pi_{i}\right) \subset \mathscr{L}^{1}(G), \forall i$; so $\pi_{i} \in \Pi_{\mathfrak{b}}^{1}, \forall i \in I$, and $c_{f}^{\pi} \times c_{f^{\prime}}^{\pi^{\prime}} \in \operatorname{Coeff}_{K}\left(\Pi_{\mathfrak{b}}^{1}\right)$. Finally, it is well-known that $\Pi_{\mathfrak{b}}^{1} \subset \Pi_{\mathfrak{b}}^{2}([25])$.

3.6. To check the algebra structure of $\operatorname{Coeff}_{K}\left(\Pi_{\mathrm{b}}\right)$, and see that it is really different from the algebra structure of finite-dimensional coefficients, let us give details for $G=\mathbf{S U}(1,1) \simeq \mathbf{S L}(2, \mathbb{R})$.

In that case, there are two possible Borel subalgebras $\mathfrak{b}_{+}$and $\mathfrak{b}_{-}$, with the corresponding families $\Pi_{\mathfrak{b}_{ \pm}}$of $\mathfrak{b}_{ \pm}$-extreme unitary representations of $G$. Following Bargmann [1], $\Pi_{\mathfrak{b}_{ \pm}}$can be labelled by $\frac{1}{2} \mathbb{N}: \Pi_{\mathfrak{b}_{ \pm}}=\left\{\pi_{m}^{ \pm} ; m \in \frac{1}{2} \mathbb{N}\right\}$; one has $\pi_{0}^{ \pm}=\pi_{0}$, the trivial representation, $\pi_{1 / 2}^{ \pm}$the mock discrete series representations, $\Pi_{b \pm}^{2}=\left\{\pi_{m}^{ \pm} ; m>\frac{1}{2}\right\}$ is the discrete series, the only nonintegrable discrete series representations are $\pi_{1}^{ \pm}$, so $\Pi_{\mathfrak{b}_{ \pm}}^{1}=\left\{\pi_{m}^{ \pm} ; m>1\right\}$. It is easy to check that $\check{\pi}_{m}^{+}=\pi_{m}^{-}$, so $\operatorname{Coeff}_{K}\left(\Pi_{\mathfrak{b}_{-}}\right)=\overline{\operatorname{Coeff}_{K}\left(\Pi_{\mathfrak{b}_{+}}\right)}$, and we can restrict to the case of $\Pi_{\mathfrak{b}_{+}}$.

The reduction Clebsch-Gordan formula for tensor product is:

$$
\pi_{m}^{+} \otimes \pi_{n}^{+}=\bigoplus_{i \in \mathbb{N}} \pi_{m+n+i}^{+} .
$$

This leads to the introduction of $C_{r}^{+}=\sum_{m \geqq r, m \in \frac{1}{2} \mathbb{N}} \operatorname{Coeff}_{K}\left(\pi_{m}\right), r \in \frac{1}{2} \mathbb{N}$. One has $C_{0}^{+}=\operatorname{Coeff}_{K}\left(\Pi_{\mathfrak{b}_{+}}\right), C_{1}^{+}=\operatorname{Coeff}_{K}\left(\Pi_{\mathfrak{b}_{+}}^{2}\right)$ and $C_{3 / 2}^{+}=\operatorname{Coeff}_{K}\left(\Pi_{\mathfrak{b}_{+}}^{1}\right)$. From (3.B), it results that $C_{r}^{+} \times C_{s}^{+} \subset C_{r+s}^{+}$, therefore $\left(C_{r}^{+}\right)_{r}$ is a strictly decreasing series of ideals of $\operatorname{Coeff}_{K}\left(\Pi_{\mathfrak{b}_{+}}\right)$. Let us consider $C^{b}=C_{0}^{+} / C_{1}^{+}$, and denote by $f^{b}$ the class of $f \in C_{0}^{+}$. Given a basis $\left\{\gamma_{i}\right\}$ of $\operatorname{Coeff}_{K}\left(\Pi_{1 / 2}^{+}\right)$, then $\left\{1^{b}, \gamma_{i}^{b}\right\}$ is a basis of $C^{b}$ by (3.5.5), the subspace spanned by $\left\{\gamma_{i}^{b}\right\}$ is an ideal, and any subspace of this infinite-dimensional ideal is also an ideal, so $C^{b}$ is not Noetherian. It results that $C_{0}^{+}=\operatorname{Coeff}_{K}\left(\Pi_{\mathfrak{b}_{+}}\right)$is not Noetherian, and, a fortiori, not finitely generated. Moreover, any element of $\operatorname{Coeff}_{K}\left(\Pi_{\mathfrak{b}_{+}}\right)$can be written as a polynomial of degree $\leqq 3$ in the coefficients of $\Pi_{1 / 2}^{+}$and 1 (see [4] for details).

Algebraically, the structure of $\operatorname{Coeff}_{K}\left(\Pi_{\mathfrak{b}_{+}}\right)$reflects the Clebsch-Gordan formula (3.B), and, analytically, it reflects the integrability properties of the representations 
involved. Most likely, the case of general $G$ is quite similar, but the structure of the Clebsch-Gordan formula is (to our knowledge) not known explicitly.

\section{Poisson Brackets and Quantization of Semisimple Poisson-Lie Groups}

4.1. Let $\mathfrak{U}$ be an Abelian algebra. $\mathfrak{U}$ is a Poisson algebra if it has a Lie algebra structure (the Poisson bracket) satisfying the Leibniz identity:

$$
\{a, b c\}=\{a, b\} c+b\{a, c\}, \quad \forall a, b, c \in \mathfrak{U} .
$$

A connected Lie group $G$ is a Poisson-Lie group if $C^{\infty}(G)$ has a Poisson structure compatible with its Hopf structure, which means the following: the Poisson bracket $\mathscr{P}$ defines a Poisson bracket $\mathscr{P}^{(2)}$ on $C^{\infty}(G \times G)=C^{\infty}(G) \widehat{\otimes} C^{\infty}(G)$ by:

$$
\mathscr{P}^{(2)}\left(f \otimes f^{\prime}, g \otimes g^{\prime}\right)=\mathscr{P}(f, g) \otimes f^{\prime} g^{\prime}+f g \otimes \mathscr{P}\left(f^{\prime}, g^{\prime}\right), \quad \forall f, f^{\prime}, g, g^{\prime} \in C^{\infty}(G) ;
$$

$\mathscr{P}$ is compatible with the Hopf structure of $C^{\infty}(G)$ if:

$$
\delta(\mathscr{P}(f, g))=\mathscr{P}^{(2)}(\delta(f), \delta(g)), \quad \forall f, g \in C^{\infty}(G)
$$

Belavin and Drinfel'd have indicated how all Poisson-Lie group structures on connected simple Lie groups can be found ([3]).

4.2. Let $\mathfrak{A}$ be an Abelian algebra, and let us consider a deformation, say $\star$, of the product of $\mathfrak{A}$, with formal parameter $t$, so that:

$$
a \star b=a b+\sum_{n \geqq 1} C_{n}(a, b) t^{n}, \quad \forall a, b \in \mathfrak{U} .
$$

Let $[a, b]_{\star}=a \star b-b \star a$, and $\operatorname{ad}_{\star}(a)=[a, \cdot]_{\star}$. Then $[,]_{\star}$ is a Lie deformation of the trivial Lie algebra structure of $\mathfrak{U}$, and one has:

$$
\operatorname{ad}_{\star}(a)[b \star c]=\left(\operatorname{ad}_{\star}(a) b\right) \star c+b \star\left(\operatorname{ad}_{\star}(a) c\right) \quad \forall a, b, c \in \mathfrak{A}
$$

So, if we define $\mathscr{P}(a, b)=C_{1}(a, b)-C_{1}(b, a)$, it immediately results that $\mathscr{P}$ is a Poisson bracket on $\mathfrak{A}$, and that $t^{-1}[,]_{\star}$ is a Lie deformation of the Lie algebra structure defined by this Poisson bracket.

This situation is exactly what is called a quantization of a Poisson algebra: one tries to find a deformation of the Abelian product of $\mathfrak{U}$, such that the Poisson bracket associated to that deformation (as above) is exactly the initial Poisson bracket of $\mathfrak{A}$. The most famous example of such a procedure occurs in the Weyl formalism of quantum mechanics: the Moyal product on $\mathbb{R}^{2 n}$ is a quantization, in the above sense, of the natural Poisson bracket, and quantum mechanics appears as a deformation with parameter the Planck constant, of classical mechanics (see [2]).

4.3. There is another approach to quantization: the quantization of enveloping algebras, often called "the theory of quantum groups" ([10b]). The fact that quantum groups and quantization theory of Poisson-Lie groups are dual theories is an 
intuitive belief of a lot of authors, that we shall now rigorously prove using the results of [5,6 and 11], at least for semisimple groups. Let us give some details:

4.3.1. Given a Lie bialgebra $\mathfrak{g}$, with cocycle $\rho: \mathfrak{g} \rightarrow \mathfrak{g} \wedge \mathfrak{g}$, following [11], a quantization of $(\mathfrak{g}, \rho)$ is a Hopf $\mathbb{C}[[t]]$-algebra $\mathscr{U}_{t}$ such that:

1. $\mathscr{U}_{t} \simeq \mathscr{U}(\mathfrak{g})[[t]]$ as $\mathbb{C}[[t]]$-modules, and $\mathscr{U}_{t}$ is a Hopf deformation of $\mathscr{U}(\mathfrak{g})$;

2. if $\widetilde{\Delta}$ is the coproduct of $\mathscr{U}_{t}$, for any $X \in \mathfrak{g}$, one has:

$$
\widetilde{\Delta}(X)-\widetilde{\Delta}^{\mathrm{op}}(X)=\rho(X) t+O\left(t^{2}\right) .
$$

Here, Hopf algebras have to be understood in the topological sense ([6 or 11]): for instance, $\tilde{\Delta}: \mathscr{U}_{t} \rightarrow(\mathscr{U}(\mathfrak{g}) \otimes \mathscr{U}(\mathfrak{g}))[[t]]$ and not in $\mathscr{U}_{t} \otimes \mathscr{U}_{t}$ as usual. From now on, we shall make free use of topological Hopf algebra theory, topological deformations, duality, etc. as introduced in [6]. When a topological algebra is concerned, deformation will stand for topological deformation. When purely algebraic algebras of countable dimension (e.g. $\mathscr{U}(\mathfrak{g})$ ) are concerned, we always assume that they are endowed with their natural topology ([6]); this is not exactly the same as the topological theory developed in [11], but we prefer this presentation since the topologies we introduce are t.v.s. topologies, which is not true of the topologies of [11]. Moreover, from the strict viewpoint of deformation theory, both theories are equivalent $([6])$.

The first general examples of quantization of bialgebras were given by Drinfel'd, in the case of a semisimple $g$. At the same time, he raised the following question: Can any bialgebra be quantized? By [11], the answer to Drinfel'd's question is yes for finite dimensional Lie bialgebras. Let us now check some consequences. We follow the ideas of [5 and 6]. We assume that $g$ is a finite dimensional semisimple Lie algebra. Since $\mathscr{U}=\mathscr{U}(\mathfrak{g})$ is rigid as an algebra ([10c]), given any Hopf deformation $\mathscr{U}_{t}$ of $\mathscr{U}$, then $\mathscr{U}_{t} \simeq \mathscr{U}[[t]]$, as algebras. Assume now that $\mathfrak{g}$ is a bialgebra, with cocycle $\rho$ (actually a coboundary since $\mathfrak{g}$ is semisimple), and that $\mathscr{U}_{t}$ is a quantization of $(\mathfrak{g}, \rho)$. Condition 2 above: $\widetilde{\Delta}(X)-\widetilde{\Delta}^{\mathrm{op}}(X)=\rho(X) t+$ $O\left(t^{2}\right)$ is unchanged if the product is replaced by $f \times_{\Phi} g=\Phi\left(\Phi^{-1}(f) \times \Phi^{-1}(g)\right)$, with $\Phi=\mathrm{Id}+\Phi_{1} t+O\left(t^{2}\right)$, and the coproduct by $\widetilde{\Delta}_{\Phi}=(\Phi \otimes \Phi) \circ \widetilde{\Delta} \circ \Phi^{-1}$ (i.e. under an equivalence) since $\widetilde{\Delta}_{\Phi}-\widetilde{\Delta}_{\Phi}^{\text {op }}=\widetilde{\Delta}-\widetilde{\Delta}^{\text {op }}+O\left(t^{2}\right)$. So we can assume that $\mathscr{U}_{t}=\mathscr{U}[[t]]$, as algebras. There is a little problem with the counit: let $\widetilde{\varepsilon}$ be the new counit. Following [6 and 18], $\widetilde{\varepsilon}$ is a deformation of the trivial representation of $\mathfrak{g}$, which is rigid by semisimplicity of $\mathfrak{g}$, so $\widetilde{\varepsilon}$ is the initial counit. So, only the antipode and coproduct are changed. Let us look more closely at $\widetilde{\Delta}$ : once more using semisimplicity of $\mathfrak{g}$, it was proved by Drinfel'd $[10 \mathrm{c}]$ that $\widetilde{\Delta}$ is obtained from the initial coproduct $\Delta$ by a twist, i.e.

$$
\exists \widetilde{F} \in(\mathscr{U} \otimes \mathscr{U})[[t]] / \widetilde{\Delta}=\widetilde{F} \Delta \widetilde{F}^{-1} .
$$

Now we can prove the following

4.3.2. Proposition (Theorem 2). Let $G$ be a semisimple connected Lie group, with a Poisson-Lie group structure. There exists a deformation $\star$ of the Abelian product of $C^{\infty}(G)$ such that:

1. $f \star g=f g+C_{1}(f, g) t+O\left(t^{2}\right), \quad$ with $\quad C_{1}(f, g)-C_{1}(g, f)=\{f, g\}, \forall f$, $g \in C^{\infty}(G)$ 
2. $\delta(f \star g)=\delta(f) \star \delta(g)$ (preferred deformation),

3. unit and counit are unchanged.

So the Poisson algebra $C^{\infty}(G)$ can be quantized, in the sense of (4.3), and the quantization is compatible with the coproduct $\delta$ (preferred deformation), exactly as the Poisson bracket was (we shall also use the terminology " $\star$-products" on $C^{\infty}(G)$ for preferred deformations).

Proof. Let $\rho$ be the cocycle (actually a coboundary) of $G$ into $\mathfrak{g} \wedge \mathfrak{g} \subset A(G) \widehat{\otimes} A(G)$, associated to the Poisson bracket ([10c]). Then one has:

$$
\forall f, g \in C^{\infty}(G), \forall x \in G, \quad\langle\{f, g\} \mid x\rangle=\langle f \otimes g \mid \rho(x)(x \otimes x)\rangle .
$$

Since $\rho \in C^{\infty}(G ; \mathfrak{g} \wedge \mathfrak{g})$, it extends linearly to $A(G)$, and the restriction of $\rho$ to $\mathfrak{g} \subset A(G)$ is a cocycle of $\mathfrak{g}$ (exactly the derivative of the cocycle $\rho$, since $X=$ $\left.\frac{\mathrm{d}}{\mathrm{d} \tau} \exp \tau X\right|_{\tau=0}$ in $A(G)$, for $\left.X \in \mathrm{g}_{0}\right)$, and, since $\rho$ is associated to the Poisson bracket, $(\mathfrak{g}, \rho)$ is a Lie bialgebra. From the previous discussion, thanks to [11], $(\mathfrak{g}, \rho)$ can be quantized: up to equivalence, we can assume that $\mathscr{U}_{t}=\mathscr{U}[[t]]$ as algebras, the counit is unchanged, and the new coproduct is twisted from $\Delta$, i.e.: $\widetilde{\Delta}=\widetilde{F} \Delta \widetilde{F}^{-1}$, $\widetilde{F} \in(\mathscr{U} \otimes \mathscr{U})[[t]] \subset(A(G) \widehat{\otimes} A(G))[[t]]$.

Using [5], formula $\widetilde{\Delta}=\widetilde{F} \Delta \widetilde{F}^{-1}$ defines a coassociative coproduct on $A(G)$, and there exists an extension to $A(G)$ of the new antipode of $\mathscr{U}$. So we now get a Hopf deformation, not only of $\mathscr{U}$, but in fact of $A(G)$, with unchanged product and counit.

Let us write $\widetilde{\Delta}=\Delta+\Delta_{1} t+O\left(t^{2}\right)$, the new coproduct of $A(G)$, and consider $\varsigma(S)=\Delta_{1}(S)-\Delta_{1}^{\mathrm{op}}(S)$. Then, one has:

$$
\varsigma(S \cdot T)=\Delta(S) \varsigma(T)+\varsigma(S) \Delta(T)
$$

and $\varsigma(e)=0$. By restriction to $G \subset A(G)$, it results that $\theta(x)=\varsigma(x) \Delta(x)^{-1}$ is a $C^{\infty}$ 1-cocycle from $G$ into $A(G) \widehat{\otimes} A(G)$ for the representation $\pi(x) W=\Delta(x) W \Delta(x)^{-1}$, $W \in A(G) \widehat{\otimes} A(G)$. Note that, by restriction to $\mathfrak{g} \wedge \mathfrak{g}, \pi$ is exactly the Adjoint action of $G$ on $\mathfrak{g} \wedge \mathfrak{g}$ ( $X \in \mathfrak{g} \subset A(G))$. We extend linearly $\theta$ to $A(G)$, and compute:

$$
\theta(X)=\frac{\mathrm{d}}{\mathrm{d} \tau}[\varsigma(\exp \tau X) \Delta(\exp -\tau X)]_{\tau=0}=\varsigma(X)=\rho(X), \quad \forall X \in \mathfrak{g}_{0} .
$$

So, the two $(A(G) \widehat{\otimes} A(G))$-valued 1-cocycles $\theta$ and $\rho$ of $G$ have the same derivative, therefore $\theta=\rho$ on $G$. We deduce that $\varsigma(x)=\rho(x) \Delta(x), \forall x \in G$. Then, following [6], we use $A(G)^{*}=C^{\infty}(G)$ and the duality arguments of [6], to obtain, by transposition of the new Hopf structure of $A(G)$, a new Hopf structure of $C^{\infty}(G)$, with the new product $\star={ }^{T} \tilde{\Delta}$, unchanged unit, counit and coproduct. Now: $\forall f, g \in C^{\infty}(G), \forall x \in G$,

$$
\langle f \star g-g \star f \mid x\rangle=\left\langle f \otimes g \mid\left(\tilde{\Delta}-\tilde{\Delta}^{\mathrm{op}}\right)(x)\right\rangle=\left\langle f \otimes g \mid \rho(x) \Delta(x) t+O\left(t^{2}\right)\right\rangle,
$$

so $f \star g-g \star f=\{f, g\} t+O\left(t^{2}\right)$.

\subsubsection{Remarks.}

1. From the twist formula $\widetilde{\Delta}=\widetilde{F} \Delta \widetilde{F}^{-1}, \widetilde{F} \in(\mathscr{U} \otimes \mathscr{U})[[t]]$, and (1.6), it is clear that all the cochains of the $\star$-product are bidifferential operators of finite order. 
2. Let us note that (4.3.2) is valid without any assumption on the center of $G$. The usual technical assumption " $G$ with a finite center" is not needed. This will be explained later on, in (5.3.3).

3. The proof of (4.3.2) can be applied straightforwardly to the case of doublegroups, i.e. connected Poisson-Lie groups such that the associated Lie bialgebra $(\mathfrak{g}, \rho)$ is the double of a Lie bialgebra ([20]). In fact, it is shown in [11] that a quantization of $(\mathfrak{g}, \rho)$ can be constructed with unchanged product and a new coproduct deduced from the initial one by a twist, exactly as in the semisimple case. So we can state:

4.3.3.1. Proposition (Theorem 3). Let G be a connected Poisson-Lie group such that the associated Lie bialgebra is the double of a Lie bialgebra. Then the conclusions of (4.3.2) hold for $G$.

4. There are three essential arguments in the proof of (4.3.2):

- The first one is the existence result of [11].

- The second one (due to Drinfel'd [10c]) is the rigidity of $\mathscr{U}(\mathfrak{g})$, for semisimple $\mathfrak{g}$, together with the fact that deformed coproducts on $\mathscr{U}(\mathfrak{g})$ are obtained from the initial coproduct by twisting. Using arguments of [5], extension of the new coproduct to the whole $A(G)$ follows.

- The third one is the theory of well-behaved Hopf algebras and their deformations, developed in [6]: transposition of the new Hopf structure of $A(G)$ to $C^{\infty}(G)=A(G)^{*}$ provides the wanted quantization of the Poisson bracket on $C^{\infty}(G)$.

Now, the result of [11] is valid without any assumption on $\mathfrak{g}$. So let us try to imagine what happens if $\mathfrak{g}$ is no longer assumed to be semisimple. Then the second arguments break down: $\mathscr{U}_{t}$ is not necessarily a trivial deformation of $\mathscr{U}$, and one has no information on the relations between the initial and new coproduct. It is then not clear at all that the new product and coproduct of $\mathscr{U}$ can be extended to $A(G)$, especially if we keep in mind that $\mathscr{U}$ is not dense in $A(G)$. Admitting that it can be done, then [6] machinery can be applied, but it will provide a deformation of $C^{\infty}(G)$ with changed product, and changed coproduct (in general) so the compatibility of the Poisson bracket with the initial coproduct, will not be inherited by its $\star-$ product quantization. Therefore, direct application of the existence theorem of [11] to quantization of nonsemisimple Poisson-Lie group structures is not really clear, except if the algebra structure of $\mathscr{U}$ is not changed by quantization, as will be shown in Sect. 6.

\section{5. $\star-S t a b l e ~ S u b a l g e b r a s$}

We assume that $G$ is a connected semisimple Lie group, with finite center. Some of our results are valid without the last assumption (it will be mentioned when this is the case), but most of them need the existence of a nontrivial maximal compact subgroup $K$. We assume moreover that $G$ is a Poisson-Lie group. By (4.3.2), we introduce a preferred deformation ( $\star$-product) of $C^{\infty}(G)$, with unchanged unit, counit and coproduct, which is a quantization, in the sense of (4.1), of the Poisson bracket. 
5.1. A subalgebra $\mathfrak{H}$ of $C^{\infty}(G)$ (for the Abelian product) of $C^{\infty}(G)$ is $\star$-stable (or " $\star$ can be restricted to $\mathfrak{H}$ ") if $C_{n}(f, g) \in \mathfrak{H}, \forall f, g \in \mathfrak{H}, \forall n$, where $C_{n} \in \mathscr{L}\left(C^{\infty}(G) \widehat{\otimes} C^{\infty}(G) ; C^{\infty}(G)\right)$ are the cochains of $\star: f \star g=f g+$ $\sum_{n} C_{n}(f, g) t^{n}$.

Since one has $\{f, g\}=C_{1}(f, g)-C_{1}(g, f)$, a $\star$-stable subalgebra is necessarily a Poisson subalgebra of $C^{\infty}(G)$. Moreover, the restriction of $\star$ to $\mathfrak{H}$ is obviously a deformation (in the usual algebraic sense) of the (Abelian) algebra $\mathfrak{H}$, i.e. a quantization of the Poisson algebra $\mathfrak{H}$ in the sense of (4.1). So the determination of $\star$-stable subalgebras of $C^{\infty}(G)$ will lead, first to new examples of Poisson algebras, second to new examples of quantization of Poisson algebras. We have to mention that in many cases, the Hopf structure will be lost.

A $\star$-stable subalgebra will be called a F.R.T. core (F.R.T. is for Faddeev, Reshetikhin and Takhtajan), if it is dense in $C^{\infty}(G)$. If one knows $\star$ on a F.R.T. core, then it is known on $C^{\infty}(G)$. The most famous example is the algebra $\mathscr{H}$ of coefficients of finite-dimensional representations, when $G$ is linear: if one uses the standard Drinfel'd quantization of $\mathscr{U}(\mathfrak{g})$ for classical simple linear groups, then $\mathscr{H}$, endowed with its $\star$-product, is an algebra introduced by Faddeev, Reshetikhin and Takhtajan ([5,6 and 12]). But when $G$ is not linear, $\mathscr{H}$ is no longer a core, because of (2.8.2.3), and we will show, in this section, how other F.R.T. cores can be found.

5.2. We start the computation of the $\star$-product of coefficients by the generalization of (2.3.2) and (3.2.1). We use the notation of (4.3): the new coproduct of $\mathscr{U}$ is $\widetilde{\Delta}=\widetilde{F} \Delta \widetilde{F}^{-1}, \widetilde{F} \in(\mathscr{U} \otimes \mathscr{U})[[t]]$.

\subsubsection{Proposition.}

1. Let $\pi$ and $\pi^{\prime}$ be $C^{\infty}$ representations of $G$ in t.v.s. $V$ and $V^{\prime}, f \in \mathscr{L}_{0}(V)$, $f^{\prime} \in \mathscr{L}_{0}\left(V^{\prime}\right)$. Then:

$$
c_{f}^{\pi} \star c_{f^{\prime}}^{\pi^{\prime}}=c_{\left[\left(\pi \otimes \pi^{\prime}\right)(\widetilde{F}-1)\right] \circ\left(f \otimes f^{\prime}\right) \circ\left[\left(\pi \otimes \pi^{\prime}\right)(\widetilde{F})\right]}^{\pi \otimes},
$$

where $\pi \otimes \pi^{\prime}$ is the tensor representation of $G$ on $V \widehat{\otimes} V^{\prime}$.

2. Let $\pi$ and $\pi^{\prime}$ be admissible representations of $G$ in Banach spaces $V$ and $V^{\prime}, c_{f}^{\pi}$ and $c_{f^{\prime}}^{\pi^{\prime}}$, K-finite coefficients of $\pi$ and $\pi^{\prime}$. Then:

$$
c_{f}^{\pi} \star c_{f^{\prime}}^{\pi^{\prime}}=\left.c_{\left[\left(\pi \otimes \pi^{\prime}\right)\left(\widetilde{F}^{-1}\right)\right] \circ\left(f \otimes f^{\prime}\right) \circ\left[\left(\pi \otimes \pi^{\prime}\right)(\widetilde{F})\right]}^{\pi \otimes}\right|_{G},
$$

where $\pi \otimes \pi^{\prime}$ is the tensor representation of $G \times G$ on $V \widehat{\otimes} V^{\prime}$.

Actually, the right-hand side of both formulas may seem unclear. In fact, in both cases, it is a generalized coefficient in the sense of (2.3), and this will be explained in the proof.

Proof. We start with case 1: then $\pi \otimes \pi^{\prime}$ is a $C^{\infty}$ representation of $G \times G$, so $V \widehat{\otimes} V^{\prime}$ is a $(\mathscr{U}(\mathfrak{g}) \otimes \mathscr{U}(\mathfrak{g}))$-module, that we can extend to a formal representation of $(\mathscr{U} \otimes \mathscr{U})[[t]]$ by:

$$
\left(\pi \otimes \pi^{\prime}\right)\left(\sum_{n} X_{n} t^{n}\right)=\sum_{n}\left(\pi \otimes \pi^{\prime}\right)\left(X_{n}\right) t^{n}, \quad X_{n} \in \mathscr{U} \otimes \mathscr{U} .
$$


Writing $\tilde{F}=\sum_{n} F_{n} t^{n}, \widetilde{F}^{-1}=\sum_{n} F_{n}^{\prime} t^{n}$, one has:

$$
\left(\pi \otimes \pi^{\prime}\right)(\widetilde{F})=\sum_{n}\left(\pi \otimes \pi^{\prime}\right)\left(F_{n}\right) t^{n}, \quad\left(\pi \otimes \pi^{\prime}\right)\left(\widetilde{F}^{-1}\right)=\sum_{n}\left(\pi \otimes \pi^{\prime}\right)\left(F_{n}^{\prime}\right) t^{n},
$$

and the second is the formal inverse of the first. Then:

$$
\begin{aligned}
(\pi & \left.\otimes \pi^{\prime}\right)\left(\tilde{F}^{-1}\right) \circ\left(f \otimes f^{\prime}\right) \circ\left(\pi \otimes \pi^{\prime}\right)(\widetilde{F}) \\
& =\sum_{n}\left(\sum_{i=0}^{n}\left[\left(\pi \otimes \pi^{\prime}\right)\left(F_{i}^{\prime}\right)\right] \circ\left(f \otimes f^{\prime}\right) \circ\left[\left(\pi \otimes \pi^{\prime}\right)\left(F_{n-i}\right)\right]\right) t^{n},
\end{aligned}
$$

from which we deduce that the right-hand side of the formula in 1 is a generalized coefficient of the $C^{\infty}$ representation $\pi \otimes \pi^{\prime}$ of $G$, as defined in (2.3). Then, for $x \in G$, one has:

$$
\begin{aligned}
\left(c_{f}^{\pi} \star c_{f^{\prime}}^{\pi^{\prime}}\right)(x)= & \left\langle c_{f}^{\pi} \otimes c_{f^{\prime}}^{\pi^{\prime}} \mid \widetilde{\Delta}(x)\right\rangle \\
= & \operatorname{Tr}\left(\left(f \otimes f^{\prime}\right) \circ\left[\left(\pi \otimes \pi^{\prime}\right)(\widetilde{F})\right] \circ\left[\left(\pi \otimes \pi^{\prime}\right)(x \otimes x)\right] \circ\left[\left(\pi \otimes \pi^{\prime}\right)\left(\widetilde{F}^{-1}\right)\right]\right) \\
= & \operatorname{Tr}\left(\left[\left(\pi \otimes \pi^{\prime}\right)\left(\widetilde{F}^{-1}\right)\right] \circ\left(f \otimes f^{\prime}\right) \circ\left[\left(\pi \otimes \pi^{\prime}\right)(\widetilde{F})\right]\right. \\
& \left.\circ\left[\left(\pi \otimes \pi^{\prime}\right)(x \otimes x)\right]\right) \quad(\text { by } 2.2) \\
= & c_{\left[\left(\pi \otimes \pi^{\prime}\right)\left(\widetilde{F}^{-1}\right)\right] \circ\left(f \otimes f^{\prime}\right) \circ\left[\left(\pi \otimes \pi^{\prime}\right)(\widetilde{F})\right]}^{\pi \otimes \pi^{\prime}}(x),
\end{aligned}
$$

and this proves 1 .

Now, we prove 2 . We know by (A.11) that $\pi \otimes \pi^{\prime}$ is an admissible representation of $G \times G$, and that $\left(V \widehat{\otimes} V^{\prime}\right)_{K \times K}=V_{K} \otimes\left(V^{\prime}\right)_{K}$. The action of $\mathscr{U}(\mathfrak{g} \oplus \mathfrak{g})=$ $\mathscr{U}(\mathfrak{g}) \otimes \mathscr{U}(\mathfrak{g})$ extends formally to $(\mathscr{U}(\mathfrak{g}) \otimes \mathscr{U}(\mathfrak{g}))[[t]]$ by:

$$
\left(\pi \otimes \pi^{\prime}\right)\left(\sum_{n} X_{n} t^{n}\right)=\sum_{n}\left(\pi \otimes \pi^{\prime}\right)\left(X_{n}\right) t^{n}, \quad X_{n} \in \mathscr{U} \otimes \mathscr{U} .
$$

Therefore $\left(\pi \otimes \pi^{\prime}\right)(\widetilde{F})$ and $\left(\pi \otimes \pi^{\prime}\right)\left(\widetilde{F}^{-1}\right)$ are well-defined formal operators from $V_{K} \otimes V_{K}^{\prime}$ into itself, and the second is the formal inverse of the first.

Still using (A.11), we have $\left(V \widehat{\otimes} V^{\prime}\right)_{K \times K}^{\circledast}=V_{K}^{\circledast} \otimes V_{K}^{\prime \circledast}$, as $(\mathscr{U} \otimes \mathscr{U})$-modules. We restrict to $f=\varphi \otimes \mathbf{v}$ and $f^{\prime}=\varphi^{\prime} \otimes \mathbf{v}^{\prime}$, with $\varphi \in V_{k}^{*}, \varphi^{\prime} \in V_{k}^{\prime *}, \mathbf{v} \in V_{k}$ and $\mathbf{v}^{\prime} \in V_{k}^{\prime}$. Then, using the notations of the first part of the proof:

$$
\begin{aligned}
(\pi & \left.\otimes \pi^{\prime}\right)\left(\widetilde{F}^{-1}\right) \circ\left(f \otimes f^{\prime}\right) \circ\left(\pi \otimes \pi^{\prime}\right)(\widetilde{F}) \\
& =\sum_{n}\left(\sum_{i=0}^{n}\left[\left(\pi \otimes \pi^{\prime}\right)\left(F_{i}^{\prime}\right)\right] \circ\left(f \otimes f^{\prime}\right) \circ\left[\left(\pi \otimes \pi^{\prime}\right)\left(F_{n-i}\right)\right]\right) t^{n} .
\end{aligned}
$$

But

$$
\begin{aligned}
& {\left[\left(\pi \otimes \pi^{\prime}\right)\left(F_{i}^{\prime}\right)\right] \circ\left(f \otimes f^{\prime}\right) \circ\left[\left(\pi \otimes \pi^{\prime}\right)\left(F_{n-i}\right)\right]} \\
& \quad=\left[\left(\pi \otimes \pi^{\prime}\right)^{\vee}\left(S\left(F_{n-i}\right)\right)\left(\varphi \otimes \varphi^{\prime}\right)\right] \otimes\left[\left(\pi \otimes \pi^{\prime}\right)\left(F_{i}^{\prime}\right)\left(\mathbf{v} \otimes \mathbf{v}^{\prime}\right)\right],
\end{aligned}
$$

where $S$ is the antipode of the Hopf algebra $\mathscr{U} \otimes \mathscr{U}$, and since $V_{K}^{\circledast} \otimes\left(V^{\prime}\right)_{K}^{\circledast}$ is a $\left(\mathscr{U} \otimes \mathscr{U}\right.$ )-module for $\left(\pi \otimes \pi^{\prime}\right)^{\vee}$ (the contragredient of $\pi \otimes \pi^{\prime}$ ), we can conclude that the right-hand side of the formula in 2 is a well-defined generalized $(K \times K)$-finite coefficient of the representation $\pi \otimes \pi^{\prime}$ of $G \times G$, as defined in (A.11). 
Then we compute, exactly as in the case 1 , for $x \in G$ :

$$
\begin{aligned}
\left(c_{f}^{\pi} \star c_{f^{\prime}}^{\pi^{\prime}}\right)(x) & =\operatorname{Tr}\left(\left[\left(\pi \otimes \pi^{\prime}\right)\left(\widetilde{F}^{-1}\right)\right] \circ\left(f \otimes f^{\prime}\right) \circ\left[\left(\pi \otimes \pi^{\prime}\right)(\widetilde{F})\right] \circ\left[\left(\pi \otimes \pi^{\prime}\right)(x, x)\right]\right) \\
& =c_{\left[\left(\pi \otimes \pi^{\prime}\right)\left(\tilde{F}^{-1}\right)\right] \circ\left(f \otimes f^{\prime}\right) \circ\left[\left(\pi \otimes \pi^{\prime}\right)(\tilde{F})\right]}^{\pi \otimes \pi^{\prime}}(x, x) .
\end{aligned}
$$

\subsubsection{Remarks.}

1. When $\pi$ and $\pi^{\prime}$ are unitary admissible representations of $G,(5.2 .1 .2)$ holds also for the Hilbertian tensor product representation $\pi \otimes_{2} \pi^{\prime}$ of $G \times G$ in $V \otimes_{2} V^{\prime}$, and the proof is the same.

2. (5.2.1) has a straightforward generalization to tensor products of $n$ representations, that we shall now describe. Actually, the proof is essentially the same, but we need some notations to write the resulting formula:

Let $\mathfrak{U}$ be an algebra with a coassociative coproduct $\Delta$. Define $\mathfrak{U}^{(n)}=\bigotimes^{n} \mathfrak{U}$, and, by induction, a morphism $\Delta^{(n)}: \mathfrak{U} \rightarrow \mathfrak{U}^{(n+1)}$ by:

$$
\Delta^{(1)}=\Delta, \quad \Delta^{(n)}=\left(\operatorname{Id} \otimes \Delta^{(n-1)}\right) \circ \Delta .
$$

For instance, if $\mathfrak{U}=A(G)$, with the usual coproduct $\Delta(x)=x \otimes x(x \in G)$, one checks that $\Delta^{(n)}(x)=\bigotimes_{n+1} x$.

Assume that we start with a coproduct $\Delta$ on $\mathfrak{U}$, and consider a twisted new coproduct $\widetilde{\Delta}=\widetilde{F} \Delta \widetilde{F}^{-1}, \widetilde{F} \in \mathfrak{U} \otimes \mathfrak{U}$. Then one has:

$$
\widetilde{\Delta}^{(n)}=\widetilde{F}_{n} \Delta^{(n)} \widetilde{F}_{n}^{-1},
$$

where $\widetilde{F}_{n}$ is defined by inductively by:

$$
\widetilde{F}_{1}=\widetilde{F}, \quad \widetilde{F}_{n}=\left(1 \otimes \widetilde{F}_{n-1}\right)\left(\operatorname{Id} \otimes \Delta^{(n-1)}\right)(\widetilde{F}) .
$$

Coming back to the case of $C^{\infty}(G)$, with the Abelian product defined from coproduct $\Delta(x)=x \otimes x(x \in G)$, on $A(G)$, and $\star$-product defined from coproduct $\widetilde{\Delta}=\widetilde{F} \Delta \widetilde{F}^{-1}, \widetilde{F} \in(\mathscr{U} \otimes \mathscr{U})[[t]]$, one has, for $x \in G$ :

$$
\left\langle f_{1} \ldots f_{n} \mid x\right\rangle=\left\langle f_{1} \otimes \cdots \otimes f_{n} \mid \Delta^{(n-1)}(x)\right\rangle
$$

and, by induction:

$$
\begin{aligned}
\left\langle f_{1} \star \cdots \star f_{n} \mid x\right\rangle & =\left\langle f_{1} \otimes \cdots \otimes f_{n} \mid \widetilde{\Delta}^{(n-1)}(x)\right\rangle \\
& =\left\langle f_{1} \otimes \cdots \otimes f_{n} \mid \widetilde{F}_{n-1}(\underset{n}{\otimes x}) \widetilde{F}_{n-1}^{-1}\right\rangle,
\end{aligned}
$$

and $\widetilde{F}_{n-1} \in\left(\bigotimes^{n} \mathscr{U}\right)[[t]]$.

Now we can state the generalization of (5.2.1): Given admissible Banach representations $\pi_{1}, \ldots, \pi_{n}$ of $G, c_{f_{1}}^{\pi_{1}}, \ldots, c_{f_{n}}^{\pi_{n}} K$-finite coefficients of $\pi_{1}, \ldots, \pi_{n}$, then:

$$
c_{f_{1}}^{\pi_{1}} \star \cdots \star c_{f_{n}}^{\pi_{n}}=\left.c_{\left[\left(\pi_{1} \otimes \otimes \pi_{n}\right)\left(\tilde{F}_{n-1}^{-1}\right)\right] \circ\left(f_{1} \otimes \otimes f_{n}\right) \circ\left[\left(\pi_{1} \otimes \otimes \pi_{n}\right)\left(\tilde{F}_{n-1}\right)\right]}^{\pi_{1} \otimes}\right|_{G},
$$

i.e.: $c_{f_{1}}^{\pi_{1}} \star \cdots \star c_{f_{n}}^{\pi_{n}}$ is the restriction to $G$ of a generalized $K$-finite coefficient of the representation $\pi_{1} \otimes \cdots \otimes \pi_{n}$ of $G^{n}=G \times \cdots \times G$ ( $n$ times).

The proof is a straightforward adaptation of the proof of (5.2.1). 
5.3. We now introduce some useful $\star$-stable algebras, and deduce examples of F.R.T. cores. Note that (5.3.1), (5.3.2), and (5.3.4.1) are true without the assumption that $G$ has a finite center.

5.3.1. Proposition. Let $\mathfrak{H}$ be a subalgebra of $C^{\infty}(G)$, stable under the right and left regular representations of $\mathfrak{g}$. Then $\mathfrak{H}$ is $\star$-stable.

Example. $C^{\omega}(G)$ is a $\star$-stable subalgebra of $C^{\infty}(G)$.

Proof. Let $\widetilde{\Delta}=\widetilde{F} \Delta \widetilde{F}^{-1}, \widetilde{F} \in(\mathscr{U} \otimes \mathscr{U})[[t]]$, be the new coproduct of $A(G)$, we develop $\widetilde{F}=\sum_{n} F_{n} t^{n}, \widetilde{F}^{-1}=\sum_{n} F_{n}^{\prime} t^{n}, F_{n}, F_{n}^{\prime} \in \mathscr{U} \otimes \mathscr{U}$. Given $f, g \in \mathfrak{H}$, we compute:

$$
\forall x \in G, \quad\langle f \star g \mid x\rangle=\sum_{n}\left(\sum_{i=0}^{n}\left\langle f \otimes g \mid F_{i}(x \otimes x) F_{n-i}^{\prime}\right\rangle\right) t^{n} .
$$

So, in order to prove (5.3.1), we shall prove that, given $U, V \in \mathscr{U} \otimes \mathscr{U}$, then the function $h$ defined by $h(x)=\langle f \otimes g \mid U(x \otimes x) V\rangle(x \in G)$, is an element of $\mathfrak{H}$. We can restrict to $U=u \otimes u^{\prime}, V=v \otimes v^{\prime}, u, u^{\prime}, v, v^{\prime} \in \mathscr{U}$, and then:

$$
\forall x \in G, \quad h(x)=\left\langle f \otimes g \mid(u x v) \otimes\left(u^{\prime} x v^{\prime}\right)\right\rangle=\langle f \mid u x v\rangle\left\langle g \mid u^{\prime} x v^{\prime}\right\rangle
$$

so $h=[\boldsymbol{L}(S(u)) \boldsymbol{R}(v) f] \times\left[\boldsymbol{L}\left(S\left(u^{\prime}\right)\right) \boldsymbol{R}\left(v^{\prime}\right) g\right] \in \mathfrak{H}$.

5.3.2. Corollary. Let $H$ be a closed normal subgroup of $G$. Then $\star$ induces a $\star$-product on $C^{\infty}(G / H)$.

Proof. We identify $C^{\infty}(G / H)$ with

$$
\begin{aligned}
C_{H}^{\infty}(G) & =\left\{f \in C^{\infty}(G) \mid \boldsymbol{L}(\gamma) f=f, \forall \gamma \in H\right\} \\
& =\left\{f \in C^{\infty}(G) \mid \boldsymbol{R}(\gamma) f=f, \forall \gamma \in H\right\} .
\end{aligned}
$$

Then $C^{\infty}(G / H)$ is a closed subalgebra of $C^{\infty}(G)$, stable under the left and right regular representations of $G$, and, a fortiori, of $\mathfrak{g}$, so (5.3.1) can be applied.

5.3.3. Remark. Let $\widetilde{G}$ be the universal covering of $G$; $\widetilde{G}$ is still semisimple, but not always with a finite center. Poisson-Lie structures on $G$, or $\widetilde{G}$, are in fact the same algebraic objects: bialgebra structures on $\mathfrak{g}$ (an easy consequence of the fact that cocycles of the Adjoint representation vanish on the center of $\widetilde{G})$. By (4.3.2), any such structure has a quantization on $C^{\infty}(G)$, and also on $C^{\infty}(\widetilde{G})$. Since $G \simeq \widetilde{G} / \Gamma, \Gamma$ a discrete subgroup of the center, (5.3.2) shows that one goes from quantization on $\widetilde{G}$ to quantization on $G$, by restriction of the $\star$-product on $C^{\infty}(\widetilde{G})$ to $C^{\infty}(G)=C^{\infty}(\widetilde{G} / \Gamma)$. This explains why quantization is not dependent of the usual technical assumption: $G$ with a finite center.

\subsubsection{Proposition (Theorem 4).}

1. Let $\Pi$ be a family of $C^{\infty}$ representations of $G$, and $\mathbb{C}[\Pi]$ the subalgebra of $C^{\infty}(G)$ generated by their coefficients. Then $\mathbb{C}[\Pi]$ is $\star$-stable.

2. Let $\Pi$ be a family of $K$-finite Banach representations of $G$, and $\mathbb{C}_{K}[\Pi]$ the subalgebra of $C^{\omega}(G)$ generated by their admissible coefficients. Then $\mathbb{C}_{K}[\Pi]$ is $\star$-stable. 
Proof.

1. One can use (5.3.1), but let us give a direct proof using (5.2.1). We can assume that the trivial representation $\pi^{0}$ belongs to $\Pi$. Let $\Pi^{\otimes}=\left\{\pi_{i_{1}} \otimes \cdots \otimes \pi_{i_{n}}\right.$, $\left.\pi_{i_{j}} \in \Pi, n \in \mathbb{N}^{*}\right\}$, then, by (2.3.2), $\mathbb{C}[\Pi]=\operatorname{Coeff}\left(\Pi^{\otimes}\right)$. By (5.2.1), given coefficients $c_{f}^{\pi}$ and $c_{f^{\prime}}^{\pi^{\prime}}$, of $\pi, \pi^{\prime} \in \Pi^{\otimes}$, then $c_{f}^{\pi} \star c_{f^{\prime}}^{\pi^{\prime}}$ is a generalized coefficient of $\pi \otimes \pi^{\prime}$, which belongs to $\Pi^{\otimes}$. So $\mathbb{C}[\Pi]$ is $\star$-stable.

2. Once more, we can assume that the trivial representation $\pi^{0}$ belongs to $\Pi$. Using identities:

$$
\begin{aligned}
& \boldsymbol{L}(X) c_{\varphi \otimes \mathbf{v}}^{\pi}=c_{[\check{\pi}(X) \Phi] \otimes \mathbf{v}}^{\pi} \\
& \boldsymbol{R}(X) c_{\varphi \otimes \mathbf{v}}^{\pi}=c_{\varphi \otimes[\pi(X) \mathbf{v}]}^{\pi}
\end{aligned} \quad \forall \pi \in \Pi, \forall \varphi \in V_{\pi K}^{\circledast}, \forall \mathbf{v} \in V_{\pi K}, \quad \forall X \in \mathfrak{g}_{0},
$$

and the property that $\boldsymbol{L}(X)$ and $\mathbf{R}(X)\left(X \in \mathfrak{g}_{0}\right)$ are derivations of $C^{\infty}(G)$, we deduce, using $\pi(X)\left(V_{\pi K}\right) \subset V_{\pi K}$ and $\check{\pi}(X)\left(V_{\pi K}^{\circledast}\right) \subset V_{\pi K}^{\circledast}$, that $\mathbb{C}_{K}[\Pi]$ is stable under the left and right regular representations of $\mathfrak{g}$ on $C^{\infty}(G)$. Then, we apply (5.3.1).

5.3.5. Proposition. Let $\Pi_{i f}$ be the set of irreducible finite dimensional representations of $G . G$ is linear if and only if $\operatorname{Coeff}\left(\Pi_{i f}\right)$ is a F.R.T. core.

Proof. Since $G$ is semisimple, $\operatorname{Coeff}\left(\Pi_{i f}\right)=\mathbb{C}\left[\Pi_{f}\right)$, where $\Pi_{f}$ is the set of all finite-dimensional representations of $G$. Obviously, (5.3.4) can be applied, so $\operatorname{Coeff}\left(\Pi_{i f}\right)$ is a $\star$-stable subalgebra of $C^{\infty}(G)$. The density of $\operatorname{Coeff}\left(\Pi_{i f}\right)$ in $C^{\infty}(G)$ is achieved if and only if $G$ is linear by (2.8.2) and (2.8.3.3).

5.3.6. Proposition (Theorem 5). There exists unitary irreducible representations $\pi_{1}, \ldots, \pi_{n}$ of $G$ such that $\mathbb{C}_{K}\left[\pi_{1}, \ldots, \pi_{n}\right]$ is an F.R.T. core.

Proof. Use (3.4.4) and (5.3.4).

5.3.7. Remark. If $G$ is noncompact, the representations $\pi_{1}, \ldots, \pi_{n}$ in (5.3.6) can be obtained by the principal series induction techniques (see (3.4.3) and (3.4.4)), and there is a large freedom in their choice (see (3.4.3) and (3.4.5.1)).

5.4. In this subsection, we assume that $G$ satisfies the assumptions of (3.5), and we follow the notations of that subsection.

5.4.1. Proposition (Theorem 6). Let $\Pi_{\mathfrak{b}}$ be the set of unitary b-extreme representations of $G$ (up to equivalence) including the trivial representation $\pi^{0}$. Then $\operatorname{Coeff}_{K}\left(\Pi_{\mathfrak{b}}\right)$ is a Poisson subalgebra of $C^{\infty}(G)$ and it is $\star$-stable. If $\Pi_{\mathfrak{b}}^{1}$ is the subset of integrable elements in $\Pi_{\mathfrak{b}}$, and $\Pi_{\mathfrak{b}}^{2}$ the subset of square-integrable elements, then one has:

and

$$
\left\{\operatorname{Coeff}_{K}\left(\Pi_{\mathrm{b}}^{2}\right), \operatorname{Coeff}_{K}\left(\Pi_{\mathrm{b}}^{2}\right)\right\} \subset \operatorname{Coeff}_{K}\left(\Pi_{\mathrm{b}}^{1}\right)
$$

$$
\operatorname{Coeff}_{K}\left(\Pi_{\mathfrak{b}}^{2}\right) \star \operatorname{Coeff}_{K}\left(\Pi_{\mathfrak{b}}^{2}\right) \subset \operatorname{Coeff}_{K}\left(\Pi_{\mathfrak{b}}^{1}\right)[[t]] .
$$

Proof. It is shown in (3.5.5) that $\operatorname{Coeff}_{K}\left(\Pi_{\mathfrak{b}}\right)$ is a subalgebra of $C^{\omega}(G)$. Therefore $\mathbb{C}_{K}\left[\Pi_{\mathfrak{b}}\right]=\operatorname{Coeff}_{K}\left(\Pi_{\mathfrak{b}}\right)$, applying (5.3.4), we obtain that Coeff ${ }_{K}\left(\Pi_{\mathfrak{b}}\right)$ is $\star$-stable; it results that $\operatorname{Coeff}_{K}\left(\Pi_{\mathfrak{b}}\right)$ is a Poisson subalgebra (see (5.1)).

Given $\pi, \pi^{\prime} \in \Pi_{\mathfrak{b}}^{2}$, the Hilbertian tensor product representation $\pi \otimes \pi^{\prime}$ of $G$ is admissible and reducible into $\bigoplus_{i \in I} \pi_{i}, \pi_{i} \in \Pi_{\mathfrak{b}}((3.5 .4))$. One has $\operatorname{Coeff}_{K}\left(\pi \otimes \pi^{\prime}\right)=$ $\sum_{i \in I} \operatorname{Coeff}_{K}\left(\pi_{i}\right)$; using Schwarz inequality, each $\pi_{i} \in \Pi_{\mathfrak{b}}^{1}$. 
For $K$-finite coefficients $c_{f}^{\pi}$ and $c_{f^{\prime}}^{\pi^{\prime}}$, one has:

$$
c_{f}^{\pi} \star c_{f^{\prime}}^{\pi^{\prime}}=\left.c_{\left[\left(\pi \otimes \pi^{\prime}\right)\left(\tilde{F}^{-1}\right)\right] \circ\left(f \otimes f^{\prime}\right) \circ\left[\left(\pi \otimes \pi^{\prime}\right)(\tilde{F})\right]}^{\pi \otimes}\right|_{G} ;
$$

but, since $\pi \otimes \pi^{\prime}$ is an admissible representation of $G$, the right-hand side of the formula is actually a generalized coefficient of that representation. So $c_{f}^{\pi} \star c_{f^{\prime}}^{\pi^{\prime}} \in$ $\operatorname{Coeff}_{K}\left(\Pi_{\mathfrak{b}}^{1}\right)[[t]]$, and the last formula is proved. Then $\left[c_{f}^{\pi}, c_{f^{\prime}}^{\pi^{\prime}}\right]_{\star} \in \operatorname{Coeff}_{K}\left(\Pi_{\mathfrak{b}}^{1}\right)[[t]]$, taking the first order term, we get that $\left\{c_{f}^{\pi}, c_{f^{\prime}}^{\pi^{\prime}}\right\} \in \operatorname{Coeff}_{K}\left(\Pi_{\mathfrak{b}}^{1}\right)$.

5.4.2. Remark. The explicit description of the quantization of the Poisson algebras defined in (3.6) for $G=\mathbf{S U}(1,1)$, is carried over in [4].

\section{Preferred Quantizations of General Poisson-Lie Groups}

6.1. In the previous sections of the paper, we were mostly dealing with semisimple Poisson-Lie groups. In the present section, we shall drop the semisimplicity assumption, as was announced at the end of Sect. 4 (see (4.3.3)). The fundamental property of the Poisson-Lie bracket is compatibility with the coproduct of $C^{\infty}(G)$, so it seems reasonable to impose that quantization $\star$ of the Poisson-Lie bracket has to satisfy the same compatibility property, i.e.:

$\left(\mathrm{C}_{1}\right) \quad \delta(f \star g)=\delta(f) \star \delta(g), \quad \forall f, g \in C^{\infty}(G)$.

Using duality $A(G)=C^{\infty}(G)^{*}$, and the techniques of [6], it results, by transposition, that the new structure of $C^{\infty}(G)$ defined by $\star$, comes from a bialgebra deformation of $A(G)$, with unchanged product.

We try to construct this deformation starting with the existence result of [11], which quantizes the bialgebra structure $(\mathfrak{g}, \rho)$, associated to the Poisson bracket, into a deformation $\left(\mathscr{U}_{t}, \widetilde{\Delta}\right)$ of the Hopf structure of $\mathscr{U}(\mathfrak{g})$. To succeed, we have to extend this deformation to $A(G)$; but, assuming that this step is achieved, since it can happen that the algebra structure of $\mathscr{U}(\mathfrak{g})$ is nontrivially changed (see [11]), the finally obtained $\star$-product on $C^{\infty}(G)$ will not necessarily satisfy the compatibility property $\left(\mathrm{C}_{1}\right)$. So, we shall restrict to quantizations of $(\mathfrak{g}, \rho)$ which satisfy:

$\left(\mathrm{C}_{2}\right) \quad \mathscr{U}_{t}$ is a trivial algebra deformation of $\mathscr{U}$.

From the discussion following (4.3.1), we can therefore assume that $\mathscr{U}_{t}=\mathscr{U}[[t]]$ as a $\mathbb{C}[[t]]$-algebra. We now state our quantization of $C^{\infty}(G)$ result in that case:

6.1.1. Proposition (Theorem 1). Let $G$ be a simply connected Poisson-Lie group. We assume that the associated Lie bialgebra $(\mathfrak{g}, \rho)$ has a quantization, in the sense of [11], satisfying $\left(C_{2}\right)$. Then there exists a quantization $\star$ of the Poisson algebra $C^{\infty}(G)$ (in the sense of $(4.3)$ ) such that: $C^{\infty}(G)$

1. $f \star g=f g+C_{1}(f, g) t+O\left(t^{2}\right)$, with $C_{1}(f, g)-C_{1}(g, f)=\{f, g\}, \forall f, g \in$

2. $\delta(f \star g)=\delta(f) \star \delta(g)$ (compatibility with $\delta)$,

3. The counit of $C^{\infty}(G)$ is unchanged.

Following Gerstenhaber ([6 or 13]), such a quantization is called a preferred quantization of $C^{\infty}(G)$. 
Let us make some remarks:

1. Following [11] closely, it appears that it can happen that the unit of $C^{\infty}(G)$ is changed, i.e. that the cochains of the $\star$-product do not vanish on constants.

2. In the proof of (6.1.1), we shall show that the cochains of the $\star$-product are bidifferential operators. All of them are bidifferential of finite order.

3 . In the case of a semisimple group $G$, or in the case of a double-group, $\left(\mathrm{C}_{2}\right)$ is satisfied (see the discussion following (4.3.1)) so (6.1.1) does apply if $G$ is simply connected, and we recover partly (4.3.2) and (4.3.3.1); the explicit twist formula for the coproduct (see the discussion following (4.3.1)) is necessary in order to recover completely (4.3.2) and (4.3.3.1).

6.2. The main argument in the proof (6.1.1) is an integrability result concerning formal representations, that we shall explain in this subsection. This result is proved in [18], to which we refer for more details.

Given a Lie algebra $\mathfrak{g}$, and a space $V$, a formal representation (also called a deformation of a representation) of $\mathfrak{g}$ in $V$ is a morphism from $\mathfrak{g}$ into the algebra $\mathbf{L}(V)[[t]]$, where $\mathbf{L}(V)$ is the space of linear maps. Such a morphism extends to $\mathscr{U}(\mathfrak{g})$, and this gives the definition of the notion of formal $\mathscr{U}(\mathfrak{g})$-module. Writing $\pi=\pi_{0}+\sum_{n} \pi_{n} t^{n}$, then $\pi_{0}$ is a linear representation of $\mathfrak{g}$ (the deformed representation). Since we are mainly interested by representations in a t.v.s., we restrict in the sequel to formal representations of $g$ which are valued in $\mathscr{L}(V)[[t]],(\mathscr{L}$ means continuous).

A formal $C^{\infty}$ representation of $G$ in a bornological t.v.s. $V$ (e.g. Fréchet, or dual of reflexive Fréchet) is a morphism $\pi=\sum_{n} \pi_{n} t^{n}$ from $G$ into $\mathscr{L}(V)[[t]]$, such that:

1. $\pi_{0}$ is a $C^{\infty}$ representation of $G$ in $V$ (the deformed representation);

2. $(x, \mathbf{v}) \mapsto \pi_{n}(x) \mathbf{v}$ is a continuous map from $G \times V$ into $V, \forall n \geqq 1$.

As explained in [18], the cochains $\pi_{n}, n \geqq 1$, are related to cocycles of $G$ valued in some corresponding $C^{\infty}$ representations, for which any continuous cocycle is in fact $C^{\infty}$, so one deduces ([18]):

3. $x \mapsto \pi_{n}(x)$ is $C^{\infty}$ from $G$ into $\mathscr{L}_{c}(V)$ (topology of compact convergence), $\forall n \geqq 1$.

It results that each $\pi_{n}$ extends to $A(G)$, so $\pi=\sum_{n} \pi_{n} t^{n}$ is a continuous morphism from $A(G)$ into $\mathscr{L}_{c}(V)[[t]]$, and this will be the definition of a formal representation of $A(G)$.

Using the inclusion $\mathscr{U}(\mathfrak{g}) \subset A(G)$, we can now restrict to $\left.\pi\right|_{\mathscr{U}(\mathrm{g})}$, and get a formal representation of $\mathscr{U}(\mathfrak{g})$ (or $\mathfrak{g}$ ) in $V$, that we call the differential of $\pi$. Since $X=\left.\frac{\mathrm{d}}{\mathrm{d} \tau} \exp \tau X\right|_{\tau=0}$ in $A(G)$, for $X \in \mathfrak{g}_{0}$, one has:

$$
\pi(X)=\sum_{n} \pi_{n}(X) t^{n}, \quad \text { and } \quad \pi_{n}(X)=\left.\frac{\mathrm{d}}{\mathrm{d} \tau} \pi_{n}(\exp \tau X)\right|_{\tau=0}, \quad X \in \mathfrak{g}_{0} .
$$

A formal representation $\pi$ of $\mathfrak{g}$ in $V$ can be integrated to $G$ if there exists a formal $C^{\infty}$ representation $\tilde{\pi}$ of $G$ in $V$ such that $\pi$ is the differential of $\tilde{\pi}$, in the above sense. It is shown in [18] that formal integrability, in the simply-connected case, depends only on the integrability of the first cochain, i.e. the deformed representation. The result is stated in the case when the deformed representation is the restriction of a Banach representation to its Fréchet space of $C^{\infty}$ vectors, but this 
is only for convenience, and the result is valid for any $C^{\infty}$ representation of $G$ in a bornogical t.v.s..

We state now:

6.2.1. Proposition [18]. We assume that $G$ is simply connected. Let $\pi_{0}$ be a $C^{\infty}$ representation of $G$ (and therefore of $A(G)$ ) in a bornological t.v.s. $V$, and $\pi=\pi_{0}+\sum_{n \geqq 1} \pi_{n} t^{n}$ a formal deformation of $\pi_{0}$, as a representation of $\mathrm{g}$, with cochains $\pi_{n}(X) \in \mathscr{L}(V), \forall X \in \mathfrak{g}, \forall n \in \mathbb{N}^{*}$. Then $\pi$ can be integrated to a formal $C^{\infty}$ representation of $G$ in $V$.

Still denoting by $\pi$ the extension of the integrated representation to a formal representation of $A(G)$, and using $\frac{\mathrm{d}}{\mathrm{d} \tau} \exp (\tau X)=X \cdot \exp (\tau X)=\exp (\tau X) \cdot X, \forall X \in \mathfrak{g}_{0}$, which holds in $A(G)$, we deduce that:

$$
\frac{\mathrm{d}}{\mathrm{d} \tau} \pi(\exp \tau X)=\pi(X) \cdot \pi(\exp \tau X)=\pi(\exp \tau X) \cdot \pi(X)
$$

where $\frac{\mathrm{d}}{\mathrm{d} \tau}$ means term by term derivation in $\mathscr{L}_{c}(V)[[t]]$.

6.3. Proof of (6.1.1). We start with a quantization $\mathscr{U}_{t}$, with (formal) coproduct $\tilde{\Delta}$ satisfying $\left(\mathrm{C}_{2}\right)$. So, as mentioned previously, we can assume $\mathscr{U}_{t}=\mathscr{U}[[t]]$ as $\mathbb{C}[[t]]$ algebras. We define: $\forall F \in A(G \times G)=A(G) \widehat{\otimes} A(G), \forall X \in \mathscr{U}, \pi(X) F=\widetilde{\Delta}(X) F$. If we develop $\widetilde{\Delta}(X)=\Delta(X)+\sum_{n \geqq 1} \Delta_{n}(X) t^{n}$, we obtain:

$$
\pi(X)=\sum_{n \geqq 0} \pi_{n}(X) t^{n}, \quad \text { where } \pi_{n}(X) F=\Delta_{n}(X) F, \quad \forall n \in \mathbb{N} .
$$

This is obviously a formal representation of $\mathscr{U}$ in $A(G \times G)$, and $\pi_{n}(X) \in$ $\mathscr{L}(A(G \times G)), \quad \forall n \in \mathbb{N}, \quad \forall X \in \mathscr{U}$. We can easily define $\pi_{0}(x) \quad(x \in G)$ by: $\pi_{0}(x) F=\Delta(x) F$, and we get a $C^{\infty}$ representation of $G$ in $A(G \times G)$, with differential the representation $\pi_{0}$ of $\mathscr{U}$ defined by (6.A). $\pi_{0}$ extends to $A(G)$, and one has: $\pi_{0}(S) F=\Delta(S) F, \forall S \in A(G)$.

Applying (6.2.1), there exists a formal $C^{\infty}$ representation of $G$ (which extends to $A(G))$, that we still denote by $\pi$, with differential the representation $\pi$ of $\mathscr{U}$ defined by (6.A). In other words, $\pi$ extends from $\mathscr{U}$ to $A(G)$, and $\left.\pi\right|_{G}$ is a $C^{\infty}$ formal representation of $G$.

Now, we have to define $\widetilde{\Delta}(x)(x \in G)$. Noticing that $\widetilde{\Delta}(X)=\pi(X) 1, \forall X \in$ $\mathscr{U}$, we define $\widetilde{\Delta}(x)=\pi(x) 1,(x \in G)$. The first problem is to prove the formula: $\widetilde{\Delta}\left(x x^{\prime}\right)=\widetilde{\Delta}(x) \widetilde{\Delta}\left(x^{\prime}\right), \forall x, x^{\prime} \in G$. For that, we consider:

$$
\phi_{\tau}=\pi(\exp -\tau X)[\widetilde{\Delta}(\exp \tau X) F], \quad \forall X \in \mathfrak{g}_{0}, \quad \forall F \in A(G \times G) .
$$

Using the remark following (6.2.1), we can compute:

$$
\frac{\mathrm{d}}{\mathrm{d} \tau} \phi_{\tau}=-\pi(X) \phi_{\tau}+\pi(\exp -\tau X)\left[\left(\frac{\mathrm{d}}{\mathrm{d} \tau} \widetilde{\Delta}(\exp \tau X)\right) F\right]
$$

but $\frac{\mathrm{d}}{\mathrm{d} \tau} \widetilde{\Delta}(\exp \tau X)=\pi(X) \widetilde{\Delta}(\exp \tau X)$, and $\pi(X) \pi(\exp -\tau X)=\pi(\exp -\tau X) \pi(X)$, so we finally obtain that $\frac{\mathrm{d}}{\mathrm{d} \tau} \phi_{\tau}=0$, and since $\phi_{0}=F$, we deduce that $\phi_{\tau}=F, \forall \tau \in \mathbb{R}$, 
and then that:

$$
\forall X \in \mathfrak{g}_{0}, \forall \tau \in \mathbb{R}, \forall F \in A(G \times G), \quad \pi(\exp \tau X) F=\widetilde{\Delta}(\exp \tau X) F .
$$

So, there exists a neighbourhood $W(e)$ of $e$ in $G$ such that: $\forall x \in W(e), \forall F \in$ $A(G \times G), \pi(x) F=\widetilde{\Delta}(x) F$.

Take now $x_{1}, x_{2} \in W(e)$, and apply the previous formula to $x=x_{1}$ and $F=\widetilde{\Delta}\left(x_{2}\right) 1=\pi\left(x_{2}\right) 1$, one obtains $\widetilde{\Delta}\left(x_{1} x_{2}\right)=\widetilde{\Delta}\left(x_{1}\right) \widetilde{\Delta}\left(x_{2}\right), \forall x_{1}, x_{2} \in W(e)$. By induction, we assume that $\widetilde{\Delta}\left(x_{2} \ldots x_{n}\right)=\widetilde{\Delta}\left(x_{2}\right) \ldots \widetilde{\Delta}\left(x_{n}\right), x_{i} \in W(e)$, and apply to $F=\tilde{\Delta}$ $\left(x_{2} \ldots x_{n}\right) 1=\pi\left(x_{2} \ldots x_{n}\right) 1$, we get that $\widetilde{\Delta}\left(x_{1} \ldots x_{n}\right)=\widetilde{\Delta}\left(x_{1}\right) \ldots \widetilde{\Delta}\left(x_{n}\right), x_{i} \in W(e)$.

Since $G$ is connected, given $x, x^{\prime} \in G$, we can find $x_{i}, x_{j}^{\prime} \in W(e)$ such that $x=$ $x_{1} \ldots x_{n}$ and $x^{\prime}=x_{1}^{\prime} \ldots x_{n^{\prime}}^{\prime}$. Using the last formula, it results that: $\forall x, x^{\prime} \in G, \widetilde{\Delta}\left(x x^{\prime}\right)=$ $\widetilde{\Delta}(x) \tilde{\Delta}\left(x^{\prime}\right)$.

So we have extended $\widetilde{\Delta}$ from $\mathscr{U}$ to $G$, and formula $\widetilde{\Delta}\left(x x^{\prime}\right)=\widetilde{\Delta}(x) \widetilde{\Delta}\left(x^{\prime}\right), \forall x, x^{\prime} \in G$, holds. Moreover, since $\widetilde{\Delta}(x)=\pi(x) 1$, the map $x \mapsto \widetilde{\Delta}(x)$ is $C^{\infty}$ from $G$ into $A(G \times G)[[t]]$, and extends to a continuous linear map $\widetilde{\Delta}: A(G) \rightarrow A(G \times G)[[t]]$. From the density of $\operatorname{Span}(G)$ in $A(G)$, we deduce that $\forall S, T \in A(G), \widetilde{\Delta}(S T)=$ $\widetilde{\Delta}(S) \widetilde{\Delta}(T)$.

We have to prove now that our new coproduct $\tilde{\Delta}$ on $A(G)$ is still coassociative. We consider

$$
\psi_{1}=(\operatorname{Id} \otimes \widetilde{\Delta}) \circ \widetilde{\Delta}=\sum_{n}\left[\sum_{i=0}^{n}\left(\operatorname{Id} \otimes \Delta_{i}\right) \circ \Delta_{n-i}\right] t^{n},
$$

defined on $A(G)$. The restriction $\left.\psi_{1}\right|_{G}$ is a $C^{\infty}$ mapping from $G$ into $A(G \times G \times G)[[t]]$, and one has $\psi_{1}(S T)=\psi_{1}(S) \psi_{1}(T), \quad \forall S, T \in A(G)$. Since $\frac{\mathrm{d}}{\mathrm{d} \tau}(\exp \tau X)=X \exp \tau X$ holds in $A(G)$, for $X \in \mathfrak{g}_{0}$, one has:

$$
\forall X \in \mathfrak{g}_{0}, \forall \tau \in \mathbb{R}, \quad \frac{\mathrm{d}}{\mathrm{d} \tau}\left[\psi_{1}(\exp \tau X)\right]=\psi_{1}(X) \psi_{1}(\exp \tau X) .
$$

We can do the same thing for $\psi_{2}=(\widetilde{\Delta} \otimes \mathrm{Id}) \circ \widetilde{\Delta}$, and we obtain

$$
\forall X \in \mathfrak{g}_{0}, \forall \tau \in \mathbb{R}, \quad \frac{\mathrm{d}}{\mathrm{d} \tau}\left[\psi_{2}(\exp \tau X)\right]=\psi_{2}(X) \psi_{2}(\exp \tau X)
$$

But, $\forall X \in \mathfrak{g}_{0}, \psi_{1}(X)=\psi_{2}(X)$, because $\tilde{\Delta}$ was, from the beginning, a coassociative coproduct on $\mathscr{U}$. Now, keeping in mind that $X$ and $\exp \tau X$ do commute in $A(G)$, we compute: $\forall X \in \mathfrak{g}_{0}, \forall \tau \in \mathbb{R}$,

$$
\begin{aligned}
\frac{\mathrm{d}}{\mathrm{d} \tau}\left[\psi_{2}(\exp -\tau X) \psi_{1}(\exp \tau X)\right]= & -\psi_{2}(X) \psi_{2}(\exp -\tau X) \psi_{1}(\exp \tau X) \\
& +\psi_{2}(\exp -\tau X) \psi_{1}(X) \psi_{1}(\exp \tau X)=0
\end{aligned}
$$

At $\tau=0$, we find $\psi_{2}(1) \psi_{2}(1)=1 \otimes 1 \otimes 1$, so we conclude that

$$
\forall X \in \mathfrak{g}_{0}, \forall \tau \in \mathbb{R}, \quad \psi_{2}(\exp \tau X)=\psi_{1}(\exp \tau X) .
$$


Using once more the connectedness of $G$, and formulæ

$$
\psi_{1}(S T)=\psi_{1}(S) \psi_{1}(T), \quad \psi_{2}(S T)=\psi_{2}(S) \psi_{2}(T), \quad \forall S, T \in A(G),
$$

we obtain that $\psi_{2}(x)=\psi_{1}(x), \forall x \in G$, and then, using the density of $\operatorname{Span}(G)$ in $A(G)$, that $\psi_{2}=\psi_{1}$ on $A(G)$. Therefore $\tilde{\Delta}$ is coassociative.

$A$ priori, we have only a bialgebra deformation of $A(G)$, but it is well known that any bialgebra deformation of a Hopf algebra is in fact a Hopf deformation [13], so a new counit and new antipode do exist.

Using $C^{\infty}(G)=A(G)^{*}$, and [6] transposition machinery, we transpose the new Hopf structure of $A(G)$ to get a new Hopf structure on $C^{\infty}(G)$. Since the product of $A(G)$ is unchanged, the new Hopf structure of $C^{\infty}(G)$ is a preferred deformation of the initial one. That it is a quantization of the Poisson algebra $C^{\infty}(G)$ is proved by a straightforward application of the arguments used in the proof of (4.3.2), so we do not repeat them.

Finally, we prove that the cochains of the $\star$-product are bidifferential operators. Using $\frac{\mathrm{d}}{\mathrm{d} \tau} \exp \tau X=X \cdot \exp \tau X=(\exp \tau X) X, \forall X \in \mathfrak{g}_{0}$, we can compute:

$$
\begin{aligned}
\frac{\mathrm{d}}{\mathrm{d} \tau}\left[\Delta_{n}(\exp \tau X) \Delta(\exp -\tau X)\right]= & \sum_{i=0}^{n} \Delta_{i}(\exp \tau X) \Delta_{n-i}(X) \Delta(\exp -\tau X) \\
& -\Delta_{n}(\exp \tau X) \Delta(X) \Delta(\exp -\tau X)
\end{aligned}
$$

Since $\Delta_{0}=\Delta$, we obtain

$$
\frac{\mathrm{d}}{\mathrm{d} \tau}\left[\Delta_{n}(\exp \tau X) \Delta(\exp -\tau X)\right]=\sum_{i=0}^{n-1} \Delta_{i}(\exp \tau X) \Delta_{n-i}(X) \Delta(\exp -\tau X) .
$$

So we obtain an algorithm for the computation of $\Delta_{n}$ :

$$
\Delta_{n}(\exp X)=\sum_{i=0}^{n-1} \int_{0}^{1} \Delta_{i}(\exp \tau X) \Delta_{n-i}(X) \Delta(\exp -\tau X) \mathrm{d} \tau \cdot \Delta(\exp X) .
$$

For instance

$$
\Delta_{1}(\exp X)=\int_{0}^{1} \Delta(\exp \tau X) \Delta_{1}(X) \Delta(\exp -\tau X) \mathrm{d} \tau \cdot \Delta(\exp X)
$$

But $\Delta_{1}(X) \in \mathscr{U} \otimes \mathscr{U}$, and, for $u \in \mathscr{U} \otimes \mathscr{U}, x \in G, \Delta(x) u \Delta\left(x^{-1}\right)=\operatorname{Ad}(x) u$; since the Adjoint representation respects the filtration of $\mathscr{U} \otimes \mathscr{U}$, the integral which appears in the formula for $\Delta_{1}(\exp X)$ is an element of $(\mathscr{U} \otimes \mathscr{U})_{p_{1}}$, if $\Delta_{1}(\mathfrak{g}) \subset$ $(\mathscr{U} \otimes \mathscr{U})_{p_{1}}$. So we can write $\Delta_{1}(\exp X)=u_{1}(\exp X) \Delta(\exp X)$, with $u_{1}(\exp X) \in$ $(\mathscr{U} \otimes \mathscr{U})_{p_{1}}, \forall X \in \mathfrak{g}_{0}$.

Assume by induction the existence of $p_{n-1}, u_{0}=1, u_{1}, \ldots, u_{n-1}$ such that $\Delta_{i}(\exp X)=u_{i}(\exp X) \Delta(\exp X)$, with $\Delta_{i}(X)$ and $u_{i}(\exp X) \in(\mathscr{U} \otimes \mathscr{U})_{p_{n-1}}, 1 \leqq i \leqq$ $n-1, \forall X \in \mathrm{g}_{0}$. Using (6.B), we consider:

$$
\begin{aligned}
u_{n}(\exp X) & =\sum_{i=0}^{n-1} \int_{0}^{1} \Delta_{i}(\exp \tau X) \Delta_{n-i}(X) \Delta(\exp -\tau X) \mathrm{d} \tau \\
& =\sum_{i=0}^{n-1} \int_{0}^{1} u_{i}(\exp \tau X) \operatorname{Ad}(\exp \tau X) \Delta_{n-i}(X) \mathrm{d} \tau
\end{aligned}
$$


So, if we fix $p$ such that $\operatorname{Span}\left\{\Delta_{i}(\mathfrak{g}), 1 \leqq i \leqq n-1\right\} \subset(\mathscr{U} \otimes \mathscr{U})_{p}$, and define $p_{n}=$ $p_{n-1}+p$, we obtain that $u_{n}(\exp X) \in(\mathscr{U} \otimes \mathscr{U})_{p_{n}}, \forall X \in \mathfrak{g}_{0}$. Finally, our induction proves that there exist an increasing sequence $\left(p_{n}\right)$, a neighbourhood $W(e)$ of $e$ in $G$, and $C^{\omega}$ functions $u_{n}: W(e) \mapsto(\mathscr{U} \otimes \mathscr{U})_{p_{n}}$, with $u_{0}=1$, such that:

$$
\Delta_{n}(x)=u_{n}(x) \Delta(x), \quad \forall x \in W(e) .
$$

We now use formula $\Delta_{n}(x y)=\sum_{i=0}^{n} \Delta_{i}(x) \Delta_{n-i}(y),(x, y \in G)$. For $x, y \in W(e)$, we obtain

$$
\Delta_{n}(x y)=\sum_{i=0}^{n} u_{i}(x) \operatorname{Ad}(x)\left[u_{n-i}(y)\right] \cdot \Delta(x y) .
$$

Using connectedness of $G$, we conclude that our functions $u_{n}=\Delta_{n} \cdot \Delta^{-1}$ are $(\mathscr{U} \otimes \mathscr{U})$-valued, so the cochains are bidifferential operators.

Using the fact that the topology induced by $A(G \times G)$ on $\mathscr{U} \otimes \mathscr{U}$ is its natural topology [6], and formulæ (6.C) and (6.D), it is easily seen that, for any relatively compact opened set $\mathcal{O}$ in $G$, given $n$, there exists $p_{\mathcal{O}} \in \mathbb{N}$ such that $u_{n}(\mathcal{O}) \subset$ $(\mathscr{U} \otimes \mathscr{U})_{p \mathcal{O}}$ and $\left.u_{n}\right|_{\mathcal{O}}$ is analytic; it results that $p_{\mathcal{O}}$ is independent of $\mathcal{O}$, so finally there exists $p \in \mathbb{N}$, such that $u_{n}(G) \subset(\mathscr{U} \otimes \mathscr{U})_{p}$ and $u_{n}$ is analytic from $G$ into $(\mathscr{U} \otimes \mathscr{U})_{p}$. Therefore the cochains of the $\star$-product are analytic bidifferential operators, and (a fortiori) of finite order.

\section{Free Quantizations up to Flat Functions}

Let us assume that $G$ is a connected Poisson-Lie group. We denote by $\mathscr{P}(G)$ the closed subspace in $C^{\infty}(G)$ of functions which are flat at $e$ :

$$
f \in \mathscr{P}(G) \Leftrightarrow\langle f \mid u\rangle=0, \quad \forall u \in \mathscr{U}(\mathfrak{g}) .
$$

7.1. We introduce the space $\mathbf{T}(G)$ of formal Taylor series at $e$, as follows: we fix a neighbourhood $W$ of 0 in $\mathfrak{g}$ such that the exponential mapping is a diffeomorphism from $W$ onto $\exp (W)$. We denote by $\mathbb{C}[[\mathfrak{g}]]$ the space of formal functions on $\mathfrak{g}$, i.e. the space of formal power series on $\mathfrak{g}^{*}$ : this a Fréchet space, when endowed with product topology. We define $\mathbf{T}(G)=\mathbb{C}[[\mathfrak{g}]]$. To any $f \in C^{\infty}(G)$, we associate its (formal) Taylor series at $e, \mathscr{T}(f) \in \mathbf{T}(G)$. We obtain a continuous linear mapping $\mathscr{T}: C^{\infty}(G) \rightarrow \mathbf{T}(G)$. Using the Borel Theorem ([22]), $\mathscr{T}$ is onto; by the open mapping Theorem ([22]), $\mathscr{T}$ induces an isomorphism of t.v.s. from $C^{\infty}(G) / \mathscr{P}(G)$ onto $\mathbf{T}(G)$. We identify $C^{\infty}(G) / \mathscr{P}(G)$ and $\mathbf{T}(G)$ by this isomorphism.

Note that

$$
\mathbf{T}(G \times G)=\mathbb{C}[[\mathfrak{g} \times \mathfrak{g}]]=\mathbb{C}[[\mathfrak{g}]] \widehat{\otimes} \mathbb{C}[[\mathfrak{g}]]=\mathbf{T}(G) \widehat{\otimes} \mathbf{T}(G) .
$$

Moreover, when $f \in \mathscr{P}(G)$, then $\delta(f) \in \mathscr{P}(G \times G), S(f) \in \mathscr{P}(G)$, and $\varepsilon(f)=0$, so the Hopf structure on $C^{\infty}(G)$ defines a (quotient) Hopf structure on $\mathbf{T}(G)$. It is clear that $\mathscr{P}(G)$ is an ideal of the Poisson structure of $C^{\infty}(G)$, so one has a quotient Poisson structure on $\mathbf{T}(G)$, which is compatible with the Hopf structure, since this property is true for the Poisson structure of $C^{\infty}(G)$.

The Poisson-Hopf algebra $\mathbf{T}(G)$ is a formal version of the algebra $C^{\infty}(G)$. As a Hopf algebra, it depends only on the formal Lie group structure associated to $G$, and not on $G$ itself: when $G$ and $G^{\prime}$ are locally isomorphic, $\mathbf{T}(G)=\mathbf{T}\left(G^{\prime}\right)$. 
7.1.1. Lemma. $\mathbf{T}(G)^{*}$ and $\mathscr{U}(\mathfrak{g})$ are isomorphic, as Hopf algebras.

Proof. $\mathbf{T}(G)$ is a well-behaved Hopf algebra, so $\mathbf{T}(G)^{*}$ is also a well-behaved Hopf algebra ([6]). As t.v.s., $\mathbf{T}(G) \simeq \mathbb{C}[[g]]$, so, as a t.v.s., $\mathbf{T}(G)^{*}$ is isomorphic to a space of polynomials with the natural topology ([6]). Let $\mu: C^{\infty}(G) \rightarrow \mathbf{T}(G)=$ $C^{\infty}(G) / \mathscr{P}(G)$ be the canonical map, then ${ }^{T} \mu: \mathbf{T}(G)^{*} \rightarrow A(G)=C^{\infty}(G)^{*}$ is a continuous injection from $\mathbf{T}(G)^{*}$ onto $\mathscr{P}(G)^{\perp}=\mathscr{U}(\mathfrak{g})$. Since $\mu$ is a Hopf map, so does ${ }^{T} \mu$. To conclude, one has only to remark that the Hopf structure of $\mathscr{U}(\mathfrak{g})$ is exactly the restriction of the Hopf structure of $A(G)$.

7.2. From (7.1.1), the Hopf algebra $\mathbf{T}(G)^{*}$ being identical with $\mathscr{U}(\mathfrak{g})$ endowed with its natural topology, Hopf deformations of $\mathbf{T}(G)^{*}$ are exactly Hopf deformations of $\mathscr{U}(\mathfrak{g})$, and, since the natural topology is well-behaved, using [6], (topological) Hopf deformations of $\mathbf{T}(G)$ are in one-to-one correspondence with Hopf deformations of $\mathscr{U}(\mathfrak{g})$. So, using [11], we can conclude:

7.2.1. Proposition. There exists a Hopf deformation of $\mathbf{T}(G)$, with new product $\star$, such that

$f \star g=f g+C_{1}(f, g) t+O\left(t^{2}\right)$, with $C_{1}(f, g)-C_{1}(g, f)=\{f, g\}, \forall f, g \in \mathbf{T}(G)$.

Note that the new coproduct $\widetilde{\delta}$ is not necessarily identical to $\delta$, so the compatibility condition

$$
\delta(f \star g)=\delta(f) \star \delta(g)
$$

is not necessarily satisfied. This is what one could call a free quantization of $\mathbf{T}(G)$, or, in other words, a free quantization of $C^{\infty}(G)$ up to flat functions.

\section{Appendix A. Representations of Semisimple Lie Groups}

In this appendix, we collect several definitions and results needed in this paper; we refer to Warner [25] and Schmid [21] for more details. By t.v.s., we mean topological, locally convex and complete complex vector space. of $\mathrm{g}_{0}$.

Let $G$ be a connected Lie group, $g_{0}$ its Lie algebra, $\mathfrak{g}$ the complexification

A.1. Assume that $\pi$ is a $C^{\infty}$ representation of $G$ in a t.v.s. $V$. Using the identity

$$
C^{\infty}(G ; V)=C^{\infty}(G) \widehat{\otimes} V=\mathscr{L}(A(G) ; V),
$$

one can show ([25]) that $\pi$ lifts to a representation of $A(G)$ on $V$, and one has: $\pi(T) \in \mathscr{L}(V), \forall T \in A(G)$, and the maps $T \mapsto \pi(T) \mathbf{v}$ are continuous, $\forall \mathbf{v} \in V$. Since $\mathscr{U}(\mathfrak{g}) \subset A(G), V$ becomes a $\mathscr{U}(\mathfrak{g})$-module, and each operator $\pi(u), u \in \mathscr{U}(\mathfrak{g})$, is continuous.

A.2. Let $\pi$ be a representation of $G$ in a t.v.s. $V$ and $V_{\infty}$ be the dense subspace of $C^{\infty}$-vectors of $\pi$; given $\mathbf{v} \in V_{\infty}$, we define $\widetilde{\mathbf{v}} \in C^{\infty}(G ; V)$ by $\widetilde{\mathbf{v}}(x)=\pi(x) \mathbf{v}$, and place on $V_{\infty}$ the topology induced by $C^{\infty}(G ; V)$. Then the restriction $\pi_{\infty}$ of $\pi$ to $V_{\infty}$ is a $C^{\infty}$-representation of $G$ on $V_{\infty}$, so (A.1) can be applied.

A.3. Given a representation $\pi$ of $G$ in a Banach space $V$, let $\mathrm{W}$ be a subspace of $V_{\infty}$ which is a $\mathscr{U}(\mathfrak{g})$-submodule, then it is not true that the closure $\bar{W}$ of $W$ in $V$ is 
stable under $\pi(G)$. In order to get such a property, one has to restrict to the (dense) subspace $V_{\omega}$ of analytic vectors of $\pi$, which is also a $\mathscr{U}(\mathfrak{g})$-module; then, if $W$ is a $\mathscr{U}(\mathfrak{g})$-submodule of $V_{\omega}, \bar{W}$ is $\pi(G)$-stable. Note that the existence of nontrivial analytic vectors of $\pi$ is really specific of Banach space representations: for a more general case (even Fréchet), it can very well happen that the only analytic vector is 0 ([27]).

$A$.4. Let $\pi$ be a representation of $G$ in a t.v.s. $V$; we denote by $\breve{\pi}$ the contragredient, which is continuous on a weakly dense, closed subspace $\breve{V}$ of $V^{*}$, complete if $V^{*}$ is complete. If $V$ is reflexive, then $\breve{V}=V^{*}$. If $\pi$ is $C^{\infty}$, then $\breve{V}=V^{*}$ and $\check{\pi}$ is $C^{\infty}$.

A.5. Given two representations $\pi$ and $\pi^{\prime}$ of $G$ in t.v.s. $V$ and $V^{\prime}$, the tensor product $\pi \otimes \pi^{\prime}$ is a representation of $G \times G$ in the projective tensor product $V \widehat{\otimes} V^{\prime}$. If $\pi$ and $\pi^{\prime}$ are $C^{\infty}$ representations, then so does $\pi \otimes \pi^{\prime}$. By restriction to $G$, one gets the tensor product representation $\pi \otimes \pi^{\prime}$ of $G$. When $V$ and $V^{\prime}$ are Hilbert spaces, it is often convenient to consider the Hilbert tensor product $V \otimes_{2} V^{\prime}$, and the Hilbert tensor product representation $\pi \otimes_{2} \pi^{\prime}$ of $G \times G$ (or $G$ ); one has a continuous dense inclusion $V \widehat{\otimes} V^{\prime} \subset V \otimes_{2} V^{\prime}$.

In the sequel, we assume that $G$ is a connected semisimple Lie group, with finite center. We denote by $K$ a maximal compact subgroup, by $\mathrm{t}_{0}$ its Lie algebra, $\mathrm{t}$ the complexification of $\mathrm{t}_{0}$. We denote by $\widehat{K}$ the set of (finite-dimensional) irreducible representations of $K$; for $\delta \in \widehat{K}, \xi_{\delta}$ stands for the character of $\delta$.

A.6. Let $\pi$ be a representation of $G$ in a t.v.s. $V$; a vector $\mathbf{v}$ is $K$-finite if the linear span of its $K$-orbit is finite-dimensional. Let $V_{\delta}$ be the space of $K$-finite vectors which are isotypical of type $\delta$, defining:

$$
P_{\delta}=\operatorname{dim}(\delta) \int_{K} \overline{\xi_{\delta}(k)} \pi(k) \mathrm{d} k
$$

then $P_{\delta}$ is a continuous projection on $V_{\delta}$. It is not true in full generality that $V_{\delta}$ is contained in $V_{\infty}$, but actually it holds if $\operatorname{dim} V_{\delta}<\infty$, and, in that case, one has also $V_{\delta} \subset V_{\omega}$ when $V$ is a Banach space. We denote by $V_{K}$ the space $\sum_{\delta \in \widehat{K}} V_{\delta}$, which is dense in $V$. We shall say that $\pi$ is admissible, if $\operatorname{dim} V_{\delta}<\infty, \forall \delta$. For instance, any unitary irreducible representation, or, more generally, any T.C.I. Banach representation is admissible. When $\pi$ is an admissible Banach representation, $V_{K}$ is a $\mathscr{U}(\mathfrak{g})$-submodule of $V_{\omega}$, that we call the Harish-Chandra module associated to $\pi$. From the inclusion $V_{K} \subset V_{\omega}$, it results that submodules of $V_{K}$ correspond, by closure, to subrepresentations of $\pi$, so that $\pi$ is T.C.I. if and only if $V_{K}$ is a simple $\mathscr{U}(\mathfrak{g})$-module. Moreover, still in the Banach case, admissible representations $\pi$ and $\pi^{\prime}$ are Naimark equivalent if and only if they are infiniteismally equivalent, i.e. the $\mathscr{U}(\mathfrak{g})$-modules $V_{K}$ and $V_{K}^{\prime}$ are isomorphic.

A.7. Given a representation $\pi$ of $\mathscr{U}(\mathfrak{g})$ in a vector space $V$, we say that $\mathbf{v}$ is a $\mathrm{t}$-finite vector if $\pi(\mathscr{U}(\mathrm{t})) \mathbf{v}$ is a semisimple finite-dimensional $\mathscr{U}(\mathrm{t})$-module. Denoting by $\widehat{\mathrm{t}}$ the set of irreducible finite-dimensional representations of $\mathrm{t}$, the set, $V_{\mathrm{t}}$ of $\mathrm{t}$ finite vectors is a semisimple $\mathscr{U}(\mathrm{t})$-module, which reduces into isotypical as $V_{\mathrm{t}}=$ $\sum_{\delta \in \hat{\mathfrak{t}}} V_{\delta}$, and a $\mathscr{U}(\mathfrak{g})$-submodule. We say that $\pi$ is $\mathfrak{\mathfrak { l }}$-finite if $V=V_{\mathfrak{f}}$, and admissible 
if it is t-finite with $\operatorname{dim} V_{\delta}<\infty, \forall \delta \in \widehat{\mathfrak{f}}$. We shall also use the terminology HarishChandra module for an admissible $\mathscr{U}(\mathfrak{g})$-module.

A.8. Let $\pi$ be a Harish-Chandra module; from $V=\sum_{\delta \in \hat{\mathrm{t}}} V_{\delta}$, the algebraic dual is $V^{*}=\prod_{\delta \in \hat{\mathrm{t}}} V_{\delta}^{*}$, and the space $V_{\mathrm{t}}^{\circledast}=\left(V^{*}\right)_{\mathfrak{\mathrm { f }}}$ is $\sum_{\delta \in \hat{\mathrm{f}}} V_{\delta}^{*} . V^{*}$ is a $\mathscr{U}(\mathfrak{g})$-module for the contragredient $\check{\pi}$, and $V_{\mathfrak{f}}^{\circledast}$ is a $\mathscr{U}(\mathfrak{g})$-module, which turns out to be a HarishChandra module, that we call the dual Harish-Chandra module of $\pi$.

A.9. Let $\pi$ be an admissible $\mathscr{U}(\mathfrak{g})$-module. We say that $\pi$ is $K$-admissible (or a $K$-Harish-Chandra module) if, for and $\delta \in \widehat{\mathfrak{f}}$ such that $V_{\delta} \neq\{0\}$, then $\delta \in \widehat{K}$. By integration from $\mathfrak{f}_{0}$ to $K$ of each representation $\delta, V$ becomes a representation of $K$, which is actually a $C^{\infty}$ one, if $V$ is endowed with the direct sum $V=\sum_{\delta \in \widehat{\mathfrak{f}}} V_{\delta}$ topology. The topological dual of $V$ is exactly the algebraic dual $V^{*}$, and the space $V_{K}^{\circledast}$ of $K$-finite vectors of the contragredient representation of $K$ is exactly $V_{\mathfrak{f}}^{\circledast}$, so the dual Harish-Chandra module of $\pi$ is also $K$-admissible.

A.10. When $\pi$ is a T.C.I. Banach representation of $G$, then $V_{K}$ is an irreducible $K$-Harish-Chandra module. This useful correspondence can be reversed, in a slightly more general context than the irreducible one: actually, if $\pi$ is a $K$-admissible finite length $\mathscr{U}(\mathfrak{g})$-module on a space $V$, then $V$ is the space of $K$-finite vectors of some Banach space (or even Hilbert space) representation $\tilde{\pi}$ of $G$, and $\pi$ is the Harish-Chandra module associated to $\widetilde{\pi}$ by (A.6). Obviously, there is no unicity of $\tilde{\pi}$ if one thinks of the usual equivalence of representations of $G$, but, up to Naimark equivalence, $\tilde{\pi}$ is unique (A.6). We call such a procedure a globalization of $\pi$, following SCHMID [21], where a very elegant and complete discussion of globalizations is developed.

A.11. Let $\pi$ and $\pi^{\prime}$ be two admissible Banach representations of $G$, in spaces $V$ and $V^{\prime}$.

A.11.1. Lemma. $\pi \otimes \pi^{\prime}$ is an admissible representation of $G \times G$ in $V \widehat{\otimes} V^{\prime}$. One has

$$
\left(V \widehat{\otimes} V^{\prime}\right)_{K \times K}=V_{K} \otimes V_{K^{\prime}} \quad \text { and } \quad\left(V \widehat{\otimes} V^{\prime}\right)_{K \times K}^{\circledast}=V_{K}^{\circledast} \otimes V_{K}^{\prime \circledast} .
$$

Proof. One has $\widehat{K \times} K=\left\{\delta \otimes \delta^{\prime} ; \delta, \delta^{\prime} \in \widehat{K}\right\}$, so

Therefore

$$
P_{\delta \otimes \delta^{\prime}}=\operatorname{dim}\left(\delta \otimes \delta^{\prime}\right) \int_{K \times K} \overline{\xi_{\delta \otimes \delta^{\prime}}\left(k, k^{\prime}\right)}\left(\pi \otimes \pi^{\prime}\right)\left(k, k^{\prime}\right) \mathrm{d} k \mathrm{~d} k^{\prime}=P_{\delta} \otimes P_{\delta^{\prime}} .
$$

$$
\left(V \otimes V^{\prime}\right)_{K \times K}=\sum_{\delta, \delta^{\prime} \in \widehat{K}} P_{\delta \otimes \delta^{\prime}}\left(V \widehat{\otimes} V^{\prime}\right)=\sum_{\delta, \delta^{\prime} \in \widehat{K}} P_{\delta}(V) \otimes P_{\delta^{\prime}}\left(V^{\prime}\right)=V_{K} \otimes V_{K}^{\prime} ;
$$

it follows that $\pi \otimes \pi^{\prime}$ is an admissible representation of $G \times G$. Moreover

$$
\begin{aligned}
\left(V \widehat{\otimes} V^{\prime}\right)_{K \times K}^{\circledast} & =\left(V_{K} \otimes V_{K}^{\prime}\right)_{K \times K}^{\circledast}=\sum_{\delta, \delta^{\prime} \in \widehat{K}}\left(V_{\delta} \otimes V_{\delta^{\prime}}^{\prime}\right)^{*} \\
& =\sum_{\delta, \delta^{\prime} \in \widehat{K}} V_{\delta}^{*} \otimes V_{\delta^{\prime}}^{\prime *}=V_{K}^{\circledast} \otimes V_{K}^{\prime \circledast},
\end{aligned}
$$

as modules over $\mathscr{U}(\mathfrak{g} \otimes \mathfrak{g})=\mathscr{U}(\mathfrak{g}) \otimes \mathscr{U}(\mathfrak{g})$. 
If $\pi$ and $\pi^{\prime}$ are admissible representations in Hilbert space, the same result holds for their Hilbertian tensor product.

\section{Appendix B. A Group-Adapted $C^{\infty}$ Stone-Weierstrass Theorem}

Let $G$ be a connected Lie group. We present here a sufficient condition for the density of a subalgebra in $C^{\infty}(G)$. The assumptions are partly the same as the classical Stone-Weierstrass Theorem (which is the $C^{0}$ case), but we need to add stability under left (or right) regular representation. So the result is valid for Lie groups only.

B.1. We state the conditions for closed subalgebras of $C^{\infty}(G)$; for applications, one generally starts with an unclosed subalgebra, and tries to apply to its closure.

B.1.1. Theorem ( $C^{\infty}$ Stone-Weierstrass Theorem for Lie groups). Let $G$ be a connected Lie group, $\mathscr{S}$ a closed subalgebra of $C^{\infty}(G)$ such that

1. $\forall f \in \mathscr{S}, \bar{f} \in \mathscr{S}$;

2. $\forall x, y \in G, x \neq y, \exists f \in \mathscr{S}$ such that $f(x) \neq f(y)$;

3. $\mathscr{S}$ is stable under the left (or right) regular representation of $G$.

Then $\mathscr{S}=C^{\infty}(G)$.

Proof. Assume that $\mathscr{S}$ is stable under the left regular (right case is similar). Let $C^{0}(G)$ be the algebra of continuous functions on $G$, with the Frechet topology of uniform convergence on compact sets. Since $\mathscr{S}$ satisfies on each compact set the assumptions of the usual Stone-Weierstrass Theorem, $\mathscr{S}$ is dense in $C^{0}(G)$, therefore its orthogonal is $\{0\}$ in $C^{0}(G)^{*}$. There is an obvious inclusion of the space of $C^{\infty}$-compactly supported functions $C_{\mathfrak{c}}^{\infty}(G)$ into $C^{0}(G)^{*}$, defined by

$$
\langle f \mid g\rangle=\int_{G} f(x) g(x) \mathrm{d} x, \quad f \in C_{\mathrm{c}}^{\infty}(G), \quad g \in C^{0}(G) .
$$

So, if $f \in C_{\mathrm{c}}^{\infty}(G)$ and satisfies $\langle f \mid S\rangle=0, \forall S \in \mathscr{S}$, then $f=0$.

Consider now the orthogonal $S^{\perp}$ of $\mathscr{S}$ in $C^{\infty}(G)^{*}=A(G)$; one has $C_{\mathrm{c}}^{\infty}(G) \subset$ $A(G)$, and $\mathscr{S}^{\perp} \cap C_{\mathrm{c}}^{\infty}(G)=\{0\}$. Since $\mathscr{S}$ is stable under the left regular representation, $\mathscr{S}^{\perp}$ is stable under $\check{\boldsymbol{L}}$, and also under its extension to $A(G)$. So $\check{\boldsymbol{L}}(S) T=$ $S \cdot T \in \mathscr{S}^{\perp}, \forall S \in A(G), \forall T \in \mathscr{S}^{\perp}$, and $\mathscr{S}^{\perp}$ is a closed left ideal of $A(G)$. But $C_{\mathrm{c}}^{\infty}(G)$ is a two-sided ideal of $A(G)$, so $C_{\mathrm{c}}^{\infty}(G) \cdot \mathscr{S}^{\perp} \subset C_{\mathrm{c}}^{\infty}(G) \cap \mathscr{S}^{\perp}=\{0\}$. Let $f_{n}$ be a sequence in $C_{\mathrm{c}}^{\infty}(G)$ such that $\lim _{n} f_{n}=\delta_{e}$ in $A(G)$, then $f_{n} \cdot S=0$, $\forall S \in \mathscr{S}^{\perp}$, but $\lim _{n}\left(f_{n} \cdot S\right)=S$, so $S=0$, and $\mathscr{S}^{\perp}=\{0\}$. It results from the HahnBanach Theorem that $\overline{\mathscr{S}}=C^{\infty}(G)$, and, since $\mathscr{S}$ is closed, $\mathscr{S}=C^{\infty}(G)$.

B.2. A direct consequence is the (well-known) density of polynomial functions in $C^{\infty}\left(\mathbb{R}^{n}\right)([22])$. Many other examples of applications are given in the present paper. The Lie group structure of the manifold $G$ is essential in the proof, and we have no idea what a $C^{\infty}$ Stone-Weierstrass theorem for a general manifold could be.

Acknowledgements. We are indebted to M. Flato, who was convinced from the beginning that the arguments of [6] were convenient for the general semisimple case. We thank W Schmid, and M Semenov-Tian-Shansky for enlightening discussions. 
Note added in proofs. After this paper was accepted, appeared the preprint Quantization of Poisson algebraic Groups and Poisson homogeneous spaces, by P. Etingof and D. Kazhdan, where it is shown that, given a Poisson Lie group $G$, there always exists a free quantization, not only up to flat functions, as shown in Sect 7 of the present paper, but actually on $C^{\infty}(G)$. In the quasi-triangular case, this quantization is preferred; in any case, it is local. These results generalize our results of Sects 4 and 7 .

\section{References}

1. Bargmann, V.: Irreducible unitary representations of the Lorentz group. Ann. Math. 48, no. 3, $568-640$ (1947)

2 Bayen, F., Flato, M., Fronsdal, C., Lichnerowicz, A., Sternheimer D : Deformation theory and quantization, I \& II Ann. Phys 111, 61-110, 111-151 (1978)

3 Belavin, A.A, Drinfel'd, V : Solution of the classical Yang-Baxter equation for simple Lie algebras Funct. Anal. Appl. 16, no 3, 1-29 (1982)

4 Bidegain, F : A candidate to noncompact quantum group To appear in Letters in Mathematical Physics (1995)

5. Bidegain, F, Pinczon, G : A star-product approach to noncompact quantum group. Lett Math. Phys. 33, no. 3, 231-240 (1995)

6 Bonneau, P., Flato, M., Gerstenhaber, M, Pinczon, G.: The hidden group structure of quantum groups: Strong duality, rigidity and preferred deformations. Commun. Math Phys. 161, 125-156 (1994)

7 Chevalley, C.: Theory of Lie groups. Princeton, New Jersey: Princeton University Press, 1946

8 Connes, A., Flato, M., Sternheimer, D : Closed star-products and cyclic cohomology. Lett Math Phys 24, no. 1, 1-12 (1991). See also: Flato, M., Sternheimer, D.: Closedness of starproducts and cohomologies. In: Lie theorie and geometry Progress in Mathematics, Basel, Boston: Birkhaüser, 1995, pp 241-260

9 De Wilde, De M., Lecomte, P.: Existence of star-products and of formal deformations of the Poisson Lie Algebra of arbitrary symplectic manifolds. Lett Math. Phys 7, no. 6, 487-496 (1983)

10a. Drinfel'd, V G.: On constant, quasi-classical solutions of the Yang-Baxter quantum equation. Sov Math. Dokl. 28, no 3, 667-671 (1983)

$10 \mathrm{~b}$ Drinfel'd, V.G.: Quantum groups Proceedings of the International Congress of Mathematicians, Berkeley 1986 (1987) pp. 798-820

10c Drinfel'd, V.G.: On almost cocommutative Hopf algebras Leningrad Math J. 1, 321-342 (1990)

10d Drinfel'd, V.G : On some unsolved problems in quantum group theory Lect Notes in Math. no 1510, $1-8$ (1992)

11 Etingof, P., Kazhdan, D : Quantization of Lie bialgebras, I. Preprint, Harvard University, Cambridge MA02138 (1995)

12 Faddeev, L.D., Reshetikhin, N.Y., Takhtajan, L.A.: Quantization of Lie groups and Lie algebras. Leningrad Math J 1, 193-226 (1990)

13 Gerstenhaber, M., Schack, S.D : Algebras, bialgebras, quantum groups and algebraic deformations. Contemp Math. 134, 51-92 (1992)

14 Grothendieck, A.: Produits tensoriels topologiques et espaces nucléaires. Memoirs of the American Mathematical Society, no. 16, Providence, RI: American Mathematical Soceity, 1955

15 Gutt, S : An explicit $\star$-product on the cotangent bundle of a Lie group Lett. Math Phys 7, no. 3, 249-258 (1983)

16 Harish-Chandra: Representations of semi-simple groups, IV. Am. J. Math. 77, 743-777 (1955)

17. Helgason, S : Differential geometry and symmetric space. New York: Academic Press, 1962

18. Lesimple, M., Pinczon, G.: Deformations of representations of Lie groups and Lie algebras. J Math. Phys 34, 4251-4272 (1993)

19a. Reshetikhin, N.Yu.: Quantized universal enveloping algebras, the Yang-Baxter equation and invariants of links, I \& II. LOMI preprints E-4-87 \& E-17-87, Leningrad (1988)

19b. Reshetikhin, N.Yu: Quantization of Lie bialgebras Int. Math. Res. Notices 7, 143-151 (1992) 
20. Reshetikhin, N. Yu., Semenov-Tian-Shansky, M.A.: Quantum $R$-matrices and factorization problem, J. Geom. and Phys. 5, no. 4, 533-550 (1988)

21. Schmid, W.: Boundary value problems for group invariant differential equations. In: Elie Cartan et les mathématiciens d'aujourd'hui, Asterisque, Société Mathématique de France, pp. 311-321 (1985)

22. Trèves, F : Topological vector spaces, distributions and kernels. New York: Academic Press, 1967

23. Turaev, V.: The Yang-Baxter equation and invariants of links. Invent. Math. 92, 527-553 (1988)

24. Varadarajan, V.S.: Infinitesimal theory of representations of semi-simple Lie groups. In: Mathematical physics and applied mathematics, 5, Amsterdam: D. Reidel Publishing 1980, pp. 131-255

25. Warner, G.: Harmonic analysis on semi-simple Lie groups, I. Berlin, Heidelberg, New York: Springer, 1972

26. Weinstein, A.: Deformation quantization. Séminaire Bourbaki, no. 789, 1994

27. Wigner, D : Private communication

Communicated by A. Connes 